\title{
Observations on Natural Resource use and (6) Conservation by the Shuar in Ecuador's Cordillera del Cóndor
}

Research

\begin{abstract}
Understanding how indigenous people use and manage natural resources is essential for promoting conservation on indigenous lands. I evaluated how one Shuar indigenous community in the Cordillera del Cóndor of Ecuador use woody taxa in mature and secondary forest, cultivate swidden land, value natural resources and view changes to their environment. Field research, conducted from January February of 2003, involved ethnobotanical survey methods and interviews with 25 community members. A total of 104 plant species were classified according to ethnobotanical use. Food, forage, construction and fuel were the most common use categories for forest plants. In the swidden plot, most plants were used for food, with native trees protected among a diversity of cultivars, primarily to attract wildlife. Mining is considered the biggest threat to conservation in the Cordillera del Cóndor's proposed Shuar Protected Area and will need to be addressed by the Shuar, supporting NGOs and the Ecuadorian government.
\end{abstract}

\section{Introduction}

There is great potential for collaboration with indigenous groups on the conservation of biological diversity in neotropical forests. In land holdings alone, indigenous territories encompass more area than all the protected areas in the Amazon Basin (Fearnside 2003, Nepstad et al. 2006, Peres 1994, Schwartzman \& Zimmerman 2005), and in countries such as Ecuador these groups maintain much of the intact forest that lies outside of protected area status (Bennett et al. 2002). In the past few decades, governments have focused their attention on many of these "undeveloped" lands, leading to an exploitation of natural resources and tremendous socio-cultural change among many indigenous groups. On the other hand, indigenous territories have served as barriers to deforestation and are a focus of current conservation efforts (Schwartz- man \& Zimmerman 2005). There is clear evidence from indigenous groups like the Kayapó in Brazil, the Cofán in Ecuador, the Guaraní in Bolivia, among others, that conservationists can collaborate with indigenous people, while indigenous groups can maintain self-determination and autonomous control of natural resources (Arambiza \& Painter 2006, Borman 1999, COICA 1989, Conklin \& Graham 1995, Schwartzman \& Zimmerman 2005, Zimmerman et al. 2001), although these relationships are complex, constantly evolving and have been heavily debated (Dove 2006). Land tenure security, the ability to resolve conflicts and the internal strength of indigenous natural resource management institutions are considered to be the main factors which positively affect conservation of biological diversity of indigenous lands (Brandon 1996). Effective partnering between conservationists and indigenous people requires that indigenous groups have strong negotiation power (Schwartzman \& Zimmerman 2005), that spaces are created that allow for transparent dialogue between partners, and that mutual interests are capitalized on (Arambiza \& Painter 2006). There is a need for conservationists to engage indigenous people in participatory ways to promote both the conservation

\section{Correspondence}

Amy E. Duchelle, School of Forest Resources and Conservation, University of Florida, U.S.A.

duchelle@ufl.edu

Ethnobotany Research \& Applications 5:005-023 (2007) 
Ethnobotany Research \& Applications

of biological diversity and cultural survival on indigenous lands.

Studies in ethnobiology and Traditional Ecological Knowledge (TEK) have served as a bridge between conservation scientists and indigenous people as a way to understand how various indigenous groups relate to their natural environment and engage them to actively conserve their natural resources, through valuing traditional knowledge and management systems. Whereas ethnobiology is essentially how traditional people identify, categorize and utilize biological species (Berlin 1992), TEK is more comprehensive in its inclusion of not only local observational knowledge of species and ecosystems, but also in its focus on the practice of resource use activities and the belief system that underscores human relationships with the natural world (Berkes et al. 2000, Nadasday 1999). TEK is considered fundamentally different from scientific knowledge in its incorporation in a wide-range of rituals and cultural practices of everyday life (Berkes et al. 2000). Both ethnobiology and TEK provide scientists with ecological information and an important base for future resource management and conservation, since much native use of natural resources is inextricably tied to traditional culture (Brandon 1996, Huntington 2000). While such studies have been adapted to understand both the evolving nature of these knowledge systems and their applicability in supporting community-based conservation efforts (Phillips \& Gentry 1993, Redford \& Padoch 1992, Shanley 2004), the challenge remains in how to integrate ethnobiology and TEK into scientific knowledge systems in just and accurate ways towards applied and participatory conservation efforts (Berkes et al. 2000, Nadasday 1999).

\section{The Shuar}

The Shuar is the second largest native ethnic group in Ecuador, with a population of approximately 40,000 people distributed in more than 400 villages in the eastern Andean slopes and Amazon lowlands of southeastern Ecuador in the provinces of Pastaza, Morona-Santiago and Zamora-Chinchipe (Bennett et al. 2002, Rubenstein 2002). The Shuar territory encompasses more than $10 \%$ of Ecuador's total area. In the male-dominated Shuar society, natural resource use is traditionally divided along gender lines. Men regularly hunt with shotguns or traditional blowguns, although in some places domesticated animals have replaced wild game as the main source of protein (Bennett et al. 2002, Rudel et al. 1999). The physically challenging task of clearing forests to create gardens is generally done by men, but women are responsible for most subsequent agricultural tasks, as well as for cooking and caring for children and animals (Bennett et al. 2002). Terra firme, or upland forest, is dominant in Amazonian Ecuador, although várzea, or seasonally flooded forests, also occur. Most Shuar live below 1000 m., occupying both flooded and upland habitats (Harner 1972) with varying productivity and resource use associated with the different forest types (Descola 1994). Shamanism is still prevalent in Shuar culture, and many Shuar view certain common plants and animals as earthly manifestations of specific souls (Bennett et al. 2002, Bottasso 1976).

The Shuar are considered to be the most-highly organized indigenous group in Ecuador, under the political umbrella of the Federación Interprovincial de Centros Shuaras (FICSH), established in 1974. FICSH, the largest of the existing Shuar political organizations manages thirtyfive associations, spanning the Ecuadorian provinces of Morona-Santiago, Zamora-Chinchipe, Orellana, Pastaza and Sucumbios. The centro, or village, is the basic administrative unit of FICSH (Salazar 1981). Each centro is generally made up of 25-30 Shuar nuclear-family households, governed by local, elected council. In 2003, FICSH established a natural resources management unit, which operates alongside the Federation's political, social and health divisions. The group initially focused on land titling and supporting agroforestry initiatives, but has extended its activities to land use planning, watershed health and biodiversity conservation. Four Shuar students were supported by the Missouri Botanic Gardens and Wildlife Conservation Society from 2001 to 2003 in a training program in conservation biology for indigenous groups in Ecuador to assume leadership in this natural resource management division.

The Shuar territory includes the biologically unique Cordillera del Cóndor region. This range rises to a maximum elevation of $2800 \mathrm{~m}$. and lies to east of the main Andean chain. It juts into the upper Amazon basin, forming a portion of the border between Ecuador and Peru. The unique geological composition of steep ridges and sandstone mesas is believed to contribute to a high degree of plant endemism with possibly the "richest flora of any similarsized area anywhere in the New World" (Schulenberg \& Awbrey 1997), attracting the interest of biologists and conservation organizations worldwide (Neill 2005). Because this region was the center of the border dispute between Ecuador and Peru for the past 160 years and Shuar settlements are generally small and dispersed, there has been little development or research in the area. The last war occurred in 1995, and a peace treaty was signed in 1998. Since then, two "peace parks" have been created along the international border, which are managed jointly by the governments of Ecuador and Peru (McNeely 2003).

Colonists from the highlands, market-driven forces and local and international NGOs are beginning to play an active role in shaping the future of this area. In addition, Ecuador's population is currently growing at approximately $1.7 \%$ each year, which is one of the highest population growth rates in South America (Unicef 2006). Recent colonization by mestizos from the Ecuadorian highlands has affected the majority of lowland indigenous groups, including the Shuar, through increased deforestation and acculturation (Rudel et al. 1999). External mining, oil and 

Shuar in Ecuador's Cordillera del Cóndor

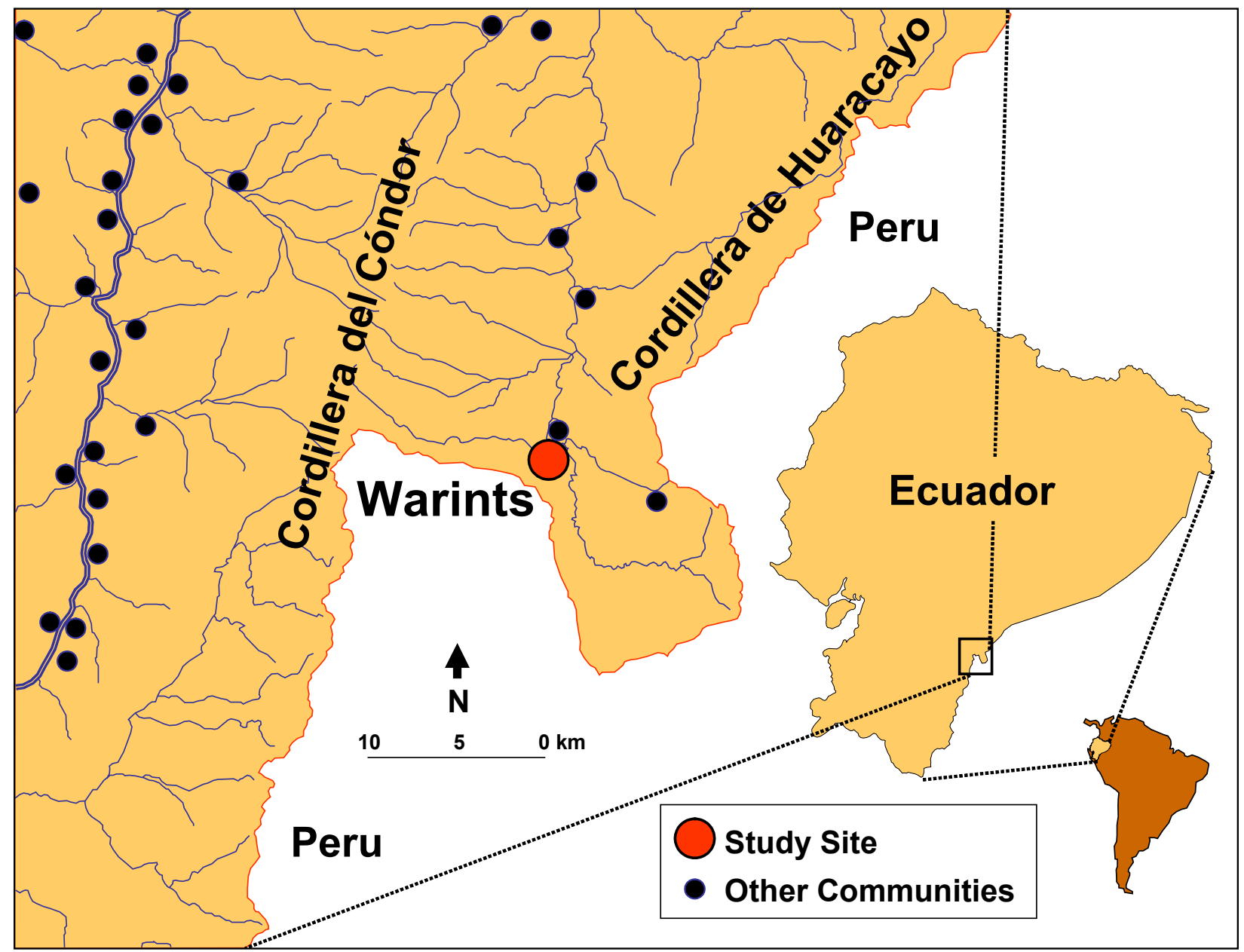

Figure 1. Study area of Warints between the Cordillera del Cóndor and Cordillera de Huaracayo in Ecuador adjacent to the Peru border.

environmental interests are also contributing to the massive changes that impact Shuar lands and culture. Many Shuar villages have clear land titles, while others do not. The Ecuadorian government retains all subsoil land rights and grants concessions to mining and oil companies, who have paid Shuar landholders large sums of money for access to explore their land.

In the past 25 years, Ecuador has increased its national parks system to a total of 33 national parks and reserves that cover nearly $20 \%$ of the country's land area (Ministerio del Ambiente 2006). The majority of these parks are inhabited by both legal and illegal occupants, making conservation within their borders a constant challenge. In the spirit of the Ecuadorian government's policy of bureaucratic devolution, the Ministry of the Environment is delegating the management of protected areas to local and regional governmental agencies. In this context, national and international non-governmental organizations (NGOs) are increasingly taking a leading role in the conservation of protected areas in Ecuador, with indigenous groups considered important participants in this effort. Of the 16 or more distinct ethnic groups in Ecuador, half of these live in
Amazonia, many occupying the last remaining intact habitats that lie in and around protected areas (Bennett et al. 2002). FICSH supported the creation of some kind of protected area in the Cordillera del Cóndor, but only if it was under Shuar jurisdiction and management - not part of the Ecuadorian Ministry of Environment's system of national parks and reserves (Schulenberg \& Awbrey 1997). Most recently, the Ecuadorian NGO Fundación Natura and the Missouri Botanic Garden have been working with the Shuar to create environmental management plans for 23 communities that will be part of a 160,000 ha Shuar Protected Area in the region (Neill 2005). In this context, more information is needed about Shuar natural resource use and management in Ecuador's Cordillera del Cóndor.

\section{Research Objective}

This research explores how one Shuar community in the Cordillera del Cóndor uses woody taxa in mature and secondary forest, cultivate swidden land, value natural resources and views changes to their environment. By integrating indigenous knowledge and experience into partici- 


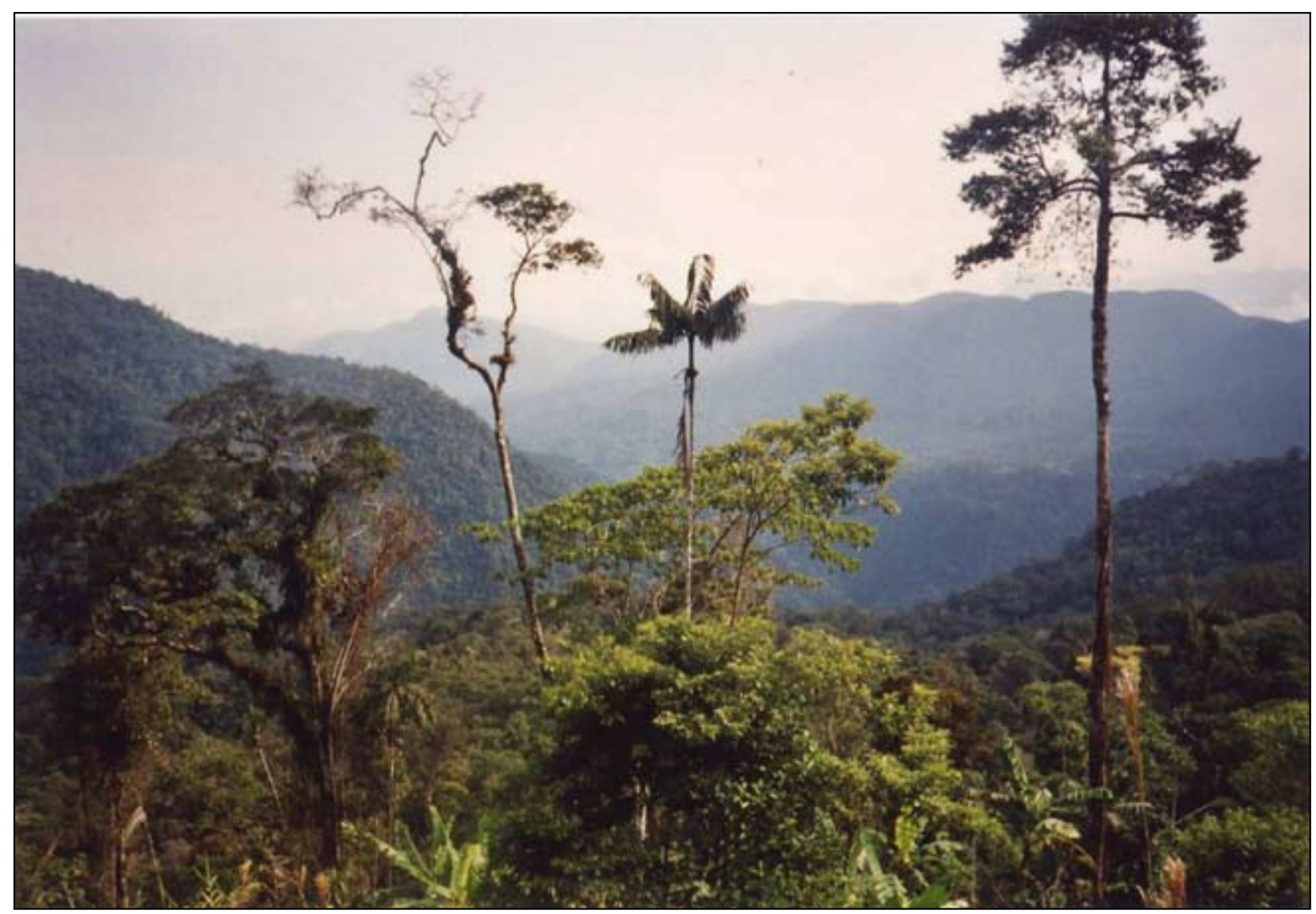

Figure 2. Study area of Warints between the Cordillera del Cóndor and Cordillera de Huaracayo in Ecuador adjacent to the Peru border.

patory environmental management plans, local needs are more likely to be met in conservation and land management decisions.

\section{Methods}

\section{Study site}

Fieldwork was conducted in the Shuar centro of Warints, located in the Coangas Watershed in the heart of the Cordillera del Cóndor (Figures 1 \& 2) from January - February of 2003 . Warints is comprised of approximately 250 Shuar people distributed among 50 households. The centro, where the airstrip and school are located, lies at 850 $\mathrm{m}$. elevation and is surrounded on three sides by steep mountains. The actual territory of Warints extends to the crest of the Cordillera del Cóndor at $2800 \mathrm{~m}$. Although the Ecuadorian government supported a small-scale copper mining company to conduct exploratory work from 19992000 on some of the adjacent slopes, about $10 \mathrm{~km}$ from the village, FICSH intervened after just over one year of operation and evicted the miners based on Shuar legislation that prohibits dialogue with mining and oil companies. The area that was explored for copper in Warints in 1999 lies at about $1800 \mathrm{~m}$. and is still visible from the centro by the large patches of exposed rock and soil that mar the previously forested mountainside. Subsistence farming and forest product extraction remain the main activities for the majority of people in Warints, with small-scale cattle ranching as a side activity for some.

\section{Ethnobotanical study and interviews with community members}

An ethnobotanical study was performed to evaluate how members of one Shuar community in Ecuador's Cordillera del Cóndor use woody taxa in mature and secondary forest, and cultivate swidden land. An initial transect (A) of $250 \mathrm{~m}$. by $4 \mathrm{~m}$. (1/10 ha) was established in mature forest at an elevation of approximately $980 \mathrm{~m}$. Specimens were collected from all native tree species over $10 \mathrm{~cm}$ in diameter at breast height ( $\mathrm{dbh}$ ) and information was gathered on scientific name, common name in Shuar, diameter, height and ethnobotanical use. Four samples of each specimen were collected; two for the National Herbarium of Ecuador, a third for the University of Wisconsin Herbarium and a fourth to use in follow-up interviews with residents of Warints. Specimens were then identified to species with the help of staff at the National Herbarium of Ecuador and the Wisconsin Herbarium. 


\section{Duchelle - Observations on Natural Resource Use and Conservation by the Shuar in Ecuador's Cordillera del Cóndor}

The same method was used in the second transect $(B)$ of $250 \mathrm{~m}$. by $4 \mathrm{~m}$. (1/10 ha), in an area of secondary forest also owned by the same landholder at an elevation of $1035 \mathrm{~m}$. that had been converted for the cultivation of manioc (Manihot esculenta Crantz) more than 30 years prior and had since been left to regenerate. The method of setting up two transects was chosen to limit the number of plants collected, while allowing general comparison in the ethnobotanical data gathered between mature and secondary forest.

In addition, specimens of all cultivated and native, ethnobotanically significant plants species were collected from a managed $20 \mathrm{~m}$ by $50 \mathrm{~m}$ (1/10 ha) swidden plot. A general distribution of crops and trees within this plot was depicted using an on-the-ground mapping technique taken from Selected guidelines for ethnobotanical research: a field manual (Alexiades \& Wood 1996). Tree species left to grow within the larger 1 ha swidden area, which encompassed the mapped plot, were also documented and specimens collected from each.

After plants were collected in the field, the variant of the inventory interview method outlined in Ethnobotany of the Shuar (Bennett et al. 2002) was taught to the four Shuar field assistants who were participants in the conservation biology training program, supported by the Missouri Botanic Garden and the Wildlife Conservation Society. In this technique, one sample from each specimen was shown independently to two or three other informants in order to compare the data given by the landholder with that of other members of the community. These informants were asked to provide the Shuar name of the plant, describe the plant's habit and give its ethnobotanical use. An ethnobotanical formula sheet was filled out by both the
Shuar field assistants and myself as each member of the community was interviewed. Additionally, both Transects $A$ and $B$ were revisited with an independent informant to further compare ethnobotanical information of the specimens found there. Ethnobotanical data was classified according to 19 major plant use categories (Bennett et al. 2002, see Table 1). All plants collected were either assigned to one or more of these categories or were considered to have no known ethnobotanical use based on the knowledge of the Shuar informants.

In addition, semi-structured interviews and free-listing and ranking exercises were conducted with 25 members of the community of Warints to provide insight into natural resource use and value and perceived threats to the environment. Interviews were conducted by Shuar conservation biology trainees in the Shuar language, with the results reported back to me a few hours after the interview had taken place.

\section{Results \& Discussion}

Results of this research include observations from the ethnobotanical data gathered in mature and secondary forest transects, as well as in the swidden area, along with information gathered from interviews and free-listing and ranking exercises with Shuar community members in Warints.

\section{Ethnobotanical data in mature and secondary forest transects, and swidden area}

A total of 147 plant collections were made not including duplicates. The plants collected represent a total of 38 known families, 70 genera and 104 species. They were

Table 1. Major use categories of Shuar plants after Bennett et. al. (2002).

\begin{tabular}{|l|l|l|}
\hline \multicolumn{2}{|l|}{ Use Categories } & Use Descriptions \\
\hline CM & Commercial & $\begin{array}{l}\text { Have monetary value outside the } \\
\text { home }\end{array}$ \\
\hline CN & Construction & $\begin{array}{l}\text { Timber used to make boats and } \\
\text { buildings }\end{array}$ \\
\hline CR & Craft & $\begin{array}{l}\text { Used to make baskets, hats and } \\
\text { other small hand-held items }\end{array}$ \\
\hline DP & Dye/paint & $\begin{array}{l}\text { Used to color fibers and other } \\
\text { materials, skin or hair }\end{array}$ \\
\hline FI & Fiber & $\begin{array}{l}\text { Used to make clothing, cord or } \\
\text { rope }\end{array}$ \\
\hline FO & Food & Eaten by humans \\
\hline FP & $\begin{array}{l}\text { Food } \\
\text { processing }\end{array}$ & $\begin{array}{l}\text { Used in cooking, preparing or } \\
\text { storing food }\end{array}$ \\
\hline FR & Forage & Eaten by animals \\
\hline FS & Fishing & Used in trapping or killing fish \\
\hline
\end{tabular}

\begin{tabular}{|l|l|l|}
\hline \multicolumn{2}{|l|}{ Use Categories } & Use Descriptions \\
\hline FE & Fuel & $\begin{array}{l}\text { Used for firewood and other non- } \\
\text { wood combustibles }\end{array}$ \\
\hline HU & Hunting & $\begin{array}{l}\text { Used in trapping or killing forest } \\
\text { game }\end{array}$ \\
\hline ME & Medicine & Used in healing \\
\hline MI & Miscellaneous & All others \\
\hline OR & Ornamental & Cultivated for aesthetic value \\
\hline PE & Personal & $\begin{array}{l}\text { Includes soaps, perfumes or } \\
\text { cosmetics }\end{array}$ \\
\hline PO & Poison & Known to be toxic \\
\hline RM & $\begin{array}{l}\text { Ritual/ } \\
\text { mythical }\end{array}$ & $\begin{array}{l}\text { Used in local ceremonies or } \\
\text { shamanic rituals }\end{array}$ \\
\hline TO & Tools & $\begin{array}{l}\text { Used to make paddles, brooms } \\
\text { or tool handles }\end{array}$ \\
\hline VE & Veterinary & Heal domestic animals \\
\hline
\end{tabular}


classified into the following life forms: trees, treelets, shrubs, sub-shrubs, herbs, or vines, as well as according to use (Table 2). As seen in Table 3. $99.1 \%$ of the specimens were identified to family, $93.3 \%$ to genus and $75 \%$ to species or species affinity.

Ethnobotanically-useful plants are categorized according to whether they were collected in mature or secondary forest, or in the swidden area. In Transect A, 19 families, 29 genera, 36 species were collected. In Transect B, 17 families, 28 genera and 35 species were identified. Forty spe-
Table 3. Number of species identified to family, genus and species and those used by the Shuar

\begin{tabular}{|l|c|c|c|}
\hline \multirow{2}{*}{ Taxa } & \multicolumn{3}{|c|}{ All species collected } \\
\cline { 2 - 4 } & Known & Unknown & Total \\
\hline Family & $103(99.1 \%)$ & $1(<1 \%)$ & $104(100 \%)$ \\
\hline Genera & $97(93.3 \%)$ & $7(6.7 \%)$ & $104(100 \%)$ \\
\hline Species & $78(75 \%)$ & $26(25 \%)$ & $104(100 \%)$ \\
\hline
\end{tabular}

Table 2. Plant species and shuar information collected in Warints.

\begin{tabular}{|c|c|c|c|c|c|c|c|}
\hline Taxa & Common name(s) & Voucher & $\begin{array}{l}\text { Forest } \\
\text { Type }^{1}\end{array}$ & Habit $^{2}$ & Status $^{3}$ & Cult? ${ }^{4}$ & Uses $^{5}$ \\
\hline \multicolumn{8}{|l|}{ ANNONACEAE } \\
\hline Rollinia mucosa (Jacq.) Baill. & Chirimoya & 135 & $\mathrm{Ag}$ & $\mathrm{h}$ & $\mathrm{y}$ & $\mathrm{y}$ & $\mathrm{FO}$ \\
\hline \multicolumn{8}{|l|}{ ARACEAE } \\
\hline Alocasia sp. & Pelma & 145 & $\mathrm{Ag}$ & $\mathrm{h}$ & $\mathrm{n}$ & $\mathrm{y}$ & FO \\
\hline Colocasia esculenta (L.) Schott & $\begin{array}{l}\text { Papachina } \\
\text { Negra/Blanca }\end{array}$ & 128,144 & $\mathrm{Ag}$ & $\mathrm{h}$ & $\mathrm{n}$ & $\mathrm{y}$ & $\mathrm{FO}$ \\
\hline \multicolumn{8}{|l|}{ ARECACEAE } \\
\hline Bactris gasipaes Kunth & Uwi, Chonta & 123 & $\mathrm{Ag}$ & $\mathrm{t}$ & $\mathrm{n}$ & $\mathrm{y}$ & FO \\
\hline Geonoma undata Klotzsch & Kapanak & 103 & $\mathrm{M}$ & $\mathrm{h}$ & $\mathrm{y}$ & - & $\mathrm{CR}$ \\
\hline Wettinia maynensis Spruce & Teren & 40 & M & $\mathrm{t}$ & $\mathrm{y}$ & - & CN FO \\
\hline \multicolumn{8}{|l|}{ ASTERACEAE } \\
\hline Mikania cordifolia (L.f.) Willd. & Inchinchi, Naik & 132 & $\mathrm{Ag}$ & $\mathrm{t}$ & $\mathrm{y}$ & $\mathrm{y}$ & FP \\
\hline $\begin{array}{l}\text { Piptocoma discolor } \\
\text { (Kunth) Pruski }\end{array}$ & Winchip & 96,160 & $\mathrm{~S}, \mathrm{Ag}$ & $\mathrm{t}$ & $y$ & $\mathrm{n}$ & $\begin{array}{l}\text { FU ME } \\
\text { MI }\end{array}$ \\
\hline \multicolumn{8}{|l|}{ BIGNONIACEAE } \\
\hline Crescentia cujete L. & Tsapa & 152 & $\mathrm{Ag}$ & $\mathrm{t}$ & $n$ & $\mathrm{y}$ & FP \\
\hline $\begin{array}{l}\text { Jacaranda copaia } \\
\text { (Aubl.) D. Don }\end{array}$ & Tsakatska & 16 & $\mathrm{M}$ & $\mathrm{t}$ & $\mathrm{y}$ & $\mathrm{n}$ & None \\
\hline $\begin{array}{l}\text { Jacaranda glabra (DC.) Bureau } \\
\text { \& K. Schum subsp. spectabilis }\end{array}$ & Kuiship, Tsakatska & 14 & $\mathrm{M}$ & $\mathrm{t}$ & $y$ & - & $\begin{array}{l}\text { CN FU } \\
\text { CR }\end{array}$ \\
\hline \multicolumn{8}{|l|}{ BIXACEAE } \\
\hline Bixa orellana L. & Ipiak, Achiote & 156 & $\mathrm{Ag}$ & $\mathrm{t}$ & $\mathrm{y}$ & $\mathrm{y}$ & FO DP \\
\hline \multicolumn{8}{|l|}{ BORAGINACEAE } \\
\hline Cordia hebeclada I.M. Johnst. & $\begin{array}{l}\text { Shimut, } \\
\text { Yunkuanim }\end{array}$ & 21 & $\mathrm{M}$ & $\mathrm{t}$ & $\mathrm{y}$ & - & $\begin{array}{l}\text { CN FU } \\
\mathrm{MI}\end{array}$ \\
\hline \multicolumn{8}{|l|}{ BURSERACEAE } \\
\hline $\begin{array}{l}\text { Dacryodes peruviana } \\
\text { (Loes.) J.F. Macbr. }\end{array}$ & $\begin{array}{l}\text { Kunchai, Numi } \\
\text { Kunchai, Copal }\end{array}$ & $\begin{array}{l}19,29 \\
155\end{array}$ & $\begin{array}{c}\mathrm{M}, \mathrm{S} \\
\mathrm{Ag}\end{array}$ & $\mathrm{t}$ & $y$ & - & $\begin{array}{l}\text { CN FU } \\
\text { FO FR } \\
\text { PE }\end{array}$ \\
\hline $\begin{array}{l}\text { Protium subserratum } \\
\text { (Engl.) Engl. }\end{array}$ & Chipia, Wantsun & 22 & $\mathrm{M}$ & $\mathrm{t}$ & $y$ & - & $\mathrm{CN} F \mathrm{~F}$ \\
\hline \multicolumn{8}{|l|}{ CARICACEAE } \\
\hline Carica microcarpa Jacq. & Wapai, Papaya & 140 & $\mathrm{Ag}$ & $\mathrm{t}$ & $\mathrm{y}$ & $\mathrm{y}$ & FO FR \\
\hline
\end{tabular}


Duchelle - Observations on Natural Resource Use and Conservation by the Shuar in Ecuador's Cordillera del Cóndor

\begin{tabular}{|c|c|c|c|c|c|c|c|}
\hline Taxa & Common name(s) & Voucher & $\begin{array}{c}\text { Forest } \\
\text { Type }^{1}\end{array}$ & Habit $^{2}$ & Status $^{3}$ & Cult? $^{4}$ & Uses $^{5}$ \\
\hline \multicolumn{8}{|l|}{ CARYOCARACEAE } \\
\hline $\begin{array}{l}\text { Anthodiscus klugii } \\
\text { Standl. ex Prance }\end{array}$ & Yumpink & 42,43 & M & $\mathrm{t}$ & $y$ & - & $\mathrm{CN} \mathrm{ME}$ \\
\hline \multicolumn{8}{|l|}{ CECROPIACEAE } \\
\hline $\begin{array}{l}\text { Cecropia ficifolia } \\
\text { Warb. Ex Snethl. }\end{array}$ & Su & 81 & $\mathrm{M}$ & $\mathrm{t}$ & $y$ & - & None \\
\hline Cecropia herthae Diels & Su & 57 & $S$ & $\mathrm{t}$ & $\mathrm{y}$ & $\mathrm{n}$ & None \\
\hline Cecropia palmatisecta Cuatrec. & Su & 13,41 & $\mathrm{M}$ & $\mathrm{t}$ & $y$ & $\mathrm{n}$ & $\begin{array}{l}\text { CN FR } \\
\text { FU }\end{array}$ \\
\hline Pourouma guianensis Aubl. & $\begin{array}{l}\text { Shuinia, Suir } \\
\text { shuinia, Ujukam } \\
\text { shuinia }\end{array}$ & 45 & $\mathrm{M}$ & $\mathrm{t}$ & $y$ & $\mathrm{n}$ & FO FU \\
\hline $\begin{array}{l}\text { Pourouma guianensis } \\
\text { Aubl. subsp. guianensis }\end{array}$ & $\begin{array}{l}\text { Amich Shuinia, } \\
\text { Uva de monte }\end{array}$ & 101 & $S$ & $\mathrm{t}$ & $y$ & $\mathrm{n}$ & FO FR \\
\hline $\begin{array}{l}\text { Licania octandra subsp. } \\
\text { pallida (Hook. f.) Prance }\end{array}$ & Yukunt & 59 & $S$ & $\mathrm{t}$ & $\bar{y}$ & $\mathrm{n}$ & CN FU \\
\hline \multicolumn{8}{|l|}{ CLUSIACEAE } \\
\hline \begin{tabular}{|l|} 
Tovomita weddeliana \\
Planch. \& Triana \\
\end{tabular} & Yaiyai, Tunkapnia & 39 & $M$ & $\mathrm{t}$ & $y$ & $\mathrm{~N}$ & $\mathrm{CN}$ \\
\hline $\begin{array}{l}\text { Vismia baccifera (L.) } \\
\text { Triana \& Planch. }\end{array}$ & $\begin{array}{l}\text { Ipiak Numi, } \\
\text { Ipiakmas }\end{array}$ & 95 & $S$ & $\mathrm{t}$ & $y$ & $\mathrm{n}$ & $\begin{array}{l}\text { CN FU } \\
\text { ME }\end{array}$ \\
\hline Ipomoea sp. & Apailnchi, Camote & 129 & $\mathrm{Ag}$ & $\mathrm{v}$ & & $y$ & $\mathrm{FO}$ \\
\hline \multicolumn{8}{|l|}{ CUCURBITACEAE } \\
\hline Cucurbita pepo L. & Namuk, Sapayo & 142 & $\mathrm{Ag}$ & $\mathrm{v}$ & $\mathrm{n}$ & $\mathrm{y}$ & $\mathrm{FO}$ \\
\hline Indet. 1 & $\begin{array}{l}\text { Namuk, Sapayo } \\
\text { Dulce }\end{array}$ & 141 & $\mathrm{Ag}$ & $\mathrm{v}$ & $n$ & $y$ & FO \\
\hline \multicolumn{8}{|l|}{ CYATHEACEAE } \\
\hline $\begin{array}{l}\text { Cyathea tortuosa } \\
\text { (Maxon) Domin }\end{array}$ & $\begin{array}{l}\text { Uunt Nashiship, } \\
\text { Nashishimp }\end{array}$ & 67 & $S$ & $\mathrm{t}$ & $y$ & $\mathrm{n}$ & $\begin{array}{l}\text { FR FU } \\
\text { PE }\end{array}$ \\
\hline \multicolumn{8}{|l|}{ CYPERACEAE } \\
\hline Cyperus sp. 1 & Piripri, Kurichip & 150 & $\mathrm{Ag}$ & $\mathrm{h}$ & - & $\mathrm{y}$ & ME \\
\hline Cyperus sp. 2 & \begin{tabular}{|l} 
Piripri, ljiat \\
Maschtai Piripri \\
\end{tabular} & 151 & $\mathrm{Ag}$ & $\mathrm{h}$ & - & $y$ & ME \\
\hline Cyperus sp. 3 & Piripri & 158 & $\mathrm{Ag}$ & $\mathrm{h}$ & - & $\mathrm{y}$ & ME \\
\hline Cyperus sp. 4 & - & 149 & $\mathrm{Ag}$ & $\mathrm{h}$ & - & $y$ & None \\
\hline \multicolumn{8}{|l|}{ DISCOREACEAE } \\
\hline Dioscorea trifida L. f. & Kenke & 124 & $\mathrm{Ag}$ & $\mathrm{h}$ & $\mathrm{y}$ & $\mathrm{y}$ & $\mathrm{FO}$ \\
\hline \multicolumn{8}{|l|}{ EUPHORBIACEAE } \\
\hline $\begin{array}{l}\text { Alchornea glandulosa } \\
\text { Poepp. \& Endl. }\end{array}$ & $\begin{array}{l}\text { Uunt Kantsa, } \\
\text { Kantsa }\end{array}$ & 49,87 & $\mathrm{M}, \mathrm{S}$ & $\mathrm{t}$ & $y$ & $\mathrm{n}$ & FR \\
\hline $\begin{array}{l}\text { Alchornea triplinervia } \\
\text { (Spreng.) Mull. Arg }\end{array}$ & Kantsa, Katsuarim & 62,84 & $S$ & $\mathrm{t}$ & $y$ & $\bar{n}$ & $\begin{array}{l}\text { CN FR } \\
\text { FU }\end{array}$ \\
\hline Alchornea sp. & Uunt Kantsa & 86 & $S$ & $\mathrm{t}$ & - & $\mathrm{n}$ & $\begin{array}{l}\text { CN FR } \\
\text { FU }\end{array}$ \\
\hline
\end{tabular}




\begin{tabular}{|c|c|c|c|c|c|c|c|}
\hline Taxa & Common name(s) & Voucher & $\begin{array}{l}\text { Forest } \\
\text { Type }^{1}\end{array}$ & Habit $^{2}$ & Status $^{3}$ & Cult? $^{4}$ & Uses $^{5}$ \\
\hline $\begin{array}{l}\text { Aparisthmium cordatum } \\
\text { (A. Juss.) Baill. }\end{array}$ & Natash & 18,66 & M & $\mathrm{t}$ & $y$ & - & $\begin{array}{l}\text { CN FP } \\
\text { FU }\end{array}$ \\
\hline Croton cuneatus Klotzsch & Tsempu & 30 & $\mathrm{M}$ & $s / t$ & $y$ & $\mathrm{n}$ & None \\
\hline $\begin{array}{l}\text { Hyeronima alchorneoides } \\
\text { Allemao }\end{array}$ & Kaa & 90 & $S$ & $\mathrm{t}$ & $y$ & $\mathrm{n}$ & CN ME \\
\hline $\begin{array}{l}\text { Mabea caudata Pax } \\
\text { \& K. Hoffm. }\end{array}$ & Tsachir & 89 & $S$ & $\mathrm{t}$ & & $\mathrm{n}$ & CN FR \\
\hline Manihot esculenta Crantz & Yucca, Mama & 120 & $\mathrm{Ag}$ & $\mathrm{h}$ & $\mathrm{n}$ & $\mathrm{y}$ & $\mathrm{FO}, \mathrm{RM}$ \\
\hline \multicolumn{8}{|l|}{ FABACEAE } \\
\hline $\begin{array}{l}\text { Abarema jupunba (Willd.) } \\
\text { Britton \& Killip }\end{array}$ & $\begin{array}{l}\text { Samik Numi, } \\
\text { Nusense }\end{array}$ & 92 & $S$ & $\mathrm{t}$ & $y$ & $\mathrm{n}$ & $\mathrm{FU}$ \\
\hline $\begin{array}{l}\text { Cedrelinga cateniformis } \\
\text { (Ducke) Ducke }\end{array}$ & Tsaik & 55 & $S$ & $\mathrm{t}$ & $\bar{y}$ & $\mathrm{n}$ & $\mathrm{CN}$ \\
\hline Dalbergia frutescens L.f. & $\begin{array}{l}\text { Namajcha, } \\
\text { Yamajcha, } \\
\text { Yantsantsa }\end{array}$ & $\begin{array}{l}73,77 \\
159\end{array}$ & $S$ & $\mathrm{t}$ & $y$ & $\mathrm{n}$ & $\begin{array}{l}\text { CN FR } \\
\text { FU }\end{array}$ \\
\hline Inga densiflora Benth. & $\begin{array}{l}\text { Machitora, } \\
\text { Machinius }\end{array}$ & 138 & $\mathrm{Ag}$ & $\mathrm{t}$ & $y$ & $\mathrm{n}$ & FO FU \\
\hline Inga sapindioides Willd. & Suir Saampi & 154,161 & $\mathrm{Ag}$ & $\mathrm{t}$ & $y$ & $\mathrm{n}$ & FO \\
\hline Inga thibaudiana DC & $\begin{array}{l}\text { Juicham Sampi, } \\
\text { Kashai Sampi }\end{array}$ & 80,93 & $S$ & $\mathrm{t}$ & $y$ & $\mathrm{n}$ & FO FR \\
\hline Inga sp. 1 & $\begin{array}{l}\text { Imiuk Sampi, } \\
\text { Muamua Sampi }\end{array}$ & 64 & $S$ & $\mathrm{t}$ & - & $\mathrm{n}$ & $\mathrm{FO}$ \\
\hline Inga sp. 2 & Kashi Sampi & 157 & $\mathrm{Ag}$ & $\mathrm{t}$ & - & $\mathrm{n}$ & FO \\
\hline Inga sp. 3 & Imik Sampi & 82 & $S$ & $\mathrm{t}$ & - & $\mathrm{n}$ & $\mathrm{FO}$ \\
\hline Lonchocarpus utilis A.C. Sm. & Timiu, Barbasco & 143 & $\mathrm{Ag}$ & $\mathrm{s}$ & $\mathrm{y}$ & $\mathrm{y}$ & FS \\
\hline Phaseolus sp. & Irpia, Frejol & 139 & $\mathrm{Ag}$ & $\mathrm{v}$ & - & $\mathrm{y}$ & $\mathrm{FO}$ \\
\hline Piptadenia anolidurus Barneby & Yurunts, Urunts & 85 & $\mathrm{~S}$ & $s / v$ & $\mathrm{y}$ & $\mathrm{n}$ & $\mathrm{FU}$ \\
\hline $\begin{array}{l}\text { Senna bacciliaris var. } \\
\text { benthamiana (J.F. Macbr.) } \\
\text { H.S. Irwin \& Barneby }\end{array}$ & Tampuch & 134 & $\mathrm{Ag}$ & $\mathrm{t}$ & $y$ & $\mathrm{n}$ & OR \\
\hline Tachigali sp. & Tinkishpinum & 6 & $\mathrm{M}$ & $\mathrm{t}$ & $\mathrm{y}$ & $\mathrm{n}$ & $\mathrm{CN}$ \\
\hline \multicolumn{8}{|l|}{ LACISTEMATACEAE } \\
\hline Lozania klugii (Mansf.) Mansf. & Tsentsena, Yukunt & 61.65 & $S$ & $\mathrm{t}$ & $y$ & $\mathrm{n}$ & $\begin{array}{l}\text { CN FR } \\
\text { FU }\end{array}$ \\
\hline \multicolumn{8}{|l|}{ LAURACEAE } \\
\hline $\begin{array}{l}\text { Endlicheria pyriformis } \\
\text { (Nees) Mez. }\end{array}$ & Takak, Takaku & 68 & $S$ & $\mathrm{~s}$ & $y$ & $\mathrm{n}$ & FR \\
\hline Nectandra gracilis Rohwer & Tinchi, Yamakai & 24 & $\mathrm{M}$ & $t$ & $y$ & $\mathrm{n}$ & $\begin{array}{l}\text { CN FU } \\
\text { DP }\end{array}$ \\
\hline Ocotea sp. 1 & $\begin{array}{l}\text { Puhu Tinchi, } \\
\text { Tinche } \\
\end{array}$ & 38 & $M$ & $\mathrm{t}$ & - & $\mathrm{n}$ & CN FR \\
\hline Ocotea sp. 2 & $\begin{array}{l}\text { Tinchi, Uchich } \\
\text { Tinchi }\end{array}$ & 78 & $S$ & $\mathrm{t}$ & - & $\mathrm{n}$ & CN FR \\
\hline \multicolumn{8}{|l|}{ LECYTHIDACEAE } \\
\hline Grias peruviana Miers & Apai & 27,79 & $M, S$ & $\mathrm{t}$ & $y$ & $\mathrm{n}$ & FO ME \\
\hline
\end{tabular}


Duchelle - Observations on Natural Resource Use and Conservation by the

Shuar in Ecuador's Cordillera del Cóndor

\begin{tabular}{|c|c|c|c|c|c|c|c|}
\hline Taxa & Common name(s) & Voucher & $\begin{array}{l}\text { Forest } \\
\text { Type }^{1}\end{array}$ & Habit $^{2}$ & Status $^{3}$ & Cult $^{4}$ & Uses $^{5}$ \\
\hline $\begin{array}{l}\text { Gustavia macaranensis } \\
\text { Philipson }\end{array}$ & Iniak, Sachi Iniak & 75 & $S$ & $\mathrm{t}$ & $y$ & $\mathrm{n}$ & FO \\
\hline \multicolumn{8}{|l|}{ MALVACEAE } \\
\hline Gossypium barbadense L. & Uruch, Algodon & 137 & $\mathrm{Ag}$ & $\begin{array}{l}\mathrm{sbs} / \\
\mathrm{s} / \mathrm{trlt}\end{array}$ & $y$ & $y$ & CR TO \\
\hline \multicolumn{8}{|l|}{ MELASTOMATACEAE } \\
\hline Bellucia pentamera Naudin & Tunkia, Tuunki & 94 & $\mathrm{~s}$ & $\mathrm{t}$ & $y$ & $\mathrm{n}$ & FO FR \\
\hline Miconia sp. 1 & Murak & 54 & $S$ & $\mathrm{t}$ & - & $\mathrm{n}$ & CN FU \\
\hline Miconia sp. 2 & $\begin{array}{l}\text { Chinchak, Uun } \\
\text { Chinchak }\end{array}$ & 35 & $\mathrm{M}$ & $\mathrm{t}$ & - & $\mathrm{n}$ & FR \\
\hline Miconia sp. 3 & Chinchak & 69 & $\mathrm{~s}$ & $\mathrm{t}$ & - & $\mathrm{n}$ & FR \\
\hline $\begin{array}{l}\text { Miconia trinervia (Sw.) } \\
\text { D.Don ex Loudon }\end{array}$ & Murak Chinchak & 91 & $S$ & $\mathrm{t}$ & $y$ & $\mathrm{n}$ & $\begin{array}{l}\text { CN FU } \\
\text { ME }\end{array}$ \\
\hline \multicolumn{8}{|l|}{ MONIMIACEAE } \\
\hline $\begin{array}{l}\text { Siparuna radiata (Poeep. } \\
\text { \& Endl.) A. DC }\end{array}$ & Tsunamuch & 83 & $S$ & s/trlt & $y$ & $\mathrm{n}$ & ME \\
\hline \multicolumn{8}{|l|}{ MORACEAE } \\
\hline Ficus sp. & Wampu & 58 & $S$ & $t$ & - & $\mathrm{n}$ & \begin{tabular}{|l|} 
CR FO \\
FR RM \\
\end{tabular} \\
\hline $\begin{array}{l}\text { Naucleopsis herrerensis } \\
\text { C.C. Berg }\end{array}$ & $\begin{array}{l}\text { Yuchatai Chimi, } \\
\text { Yaiyai }\end{array}$ & 20 & $\mathrm{M}$ & $\mathrm{t}$ & $y$ & $\mathrm{n}$ & None \\
\hline Perebea guianensis Aubl. & Sunkach & 10 & $\mathrm{M}$ & $\mathrm{t}$ & - & $\mathrm{n}$ & $\begin{array}{l}\text { FO FR } \\
\text { FU }\end{array}$ \\
\hline Perebea xantochyma H. Karst. & Tsakaya, Tsakana & 26 & $\mathrm{M}$ & $t$ & $\mathrm{y}$ & $\mathrm{n}$ & $\mathrm{FR}$ \\
\hline Pourouma bicolor Mart. & $\begin{array}{l}\text { Shuinia, Wjukam } \\
\text { Shuinia }\end{array}$ & 36 & M & $\mathrm{t}$ & $y$ & $\mathrm{n}$ & $\begin{array}{l}\text { FO FR } \\
\text { RM }\end{array}$ \\
\hline $\begin{array}{l}\text { Pseudolmedia laevis (Ruiz } \\
\text { \& Pav.) J.F. Macbr. } \\
\end{array}$ & Chimi & 17 & $M$ & $\mathrm{t}$ & $\mathrm{y}$ & $n$ & FO FU \\
\hline \multicolumn{8}{|l|}{ MYRISTICACEAE } \\
\hline Iryanthera juruensis Warb. & $\begin{array}{l}\text { Chikum, Tsempuik, } \\
\text { Unt Wayakish }\end{array}$ & 23 & $\mathrm{M}$ & $\mathrm{t}$ & $y$ & $\mathrm{n}$ & FO FR \\
\hline $\begin{array}{l}\text { Osteophoeum platyspermum } \\
\text { (Spruce ex A. DC.) Warb. }\end{array}$ & $\begin{array}{l}\text { Chukum, } \\
\text { Chikiump,Tsempu } \\
\end{array}$ & 46 & $\mathrm{M}$ & $\mathrm{t}$ & $y$ & $\mathrm{n}$ & $\mathrm{CN}$ \\
\hline Virola decorticans Ducke & Uunt Tsempu & 88 & $S$ & $\mathrm{t}$ & $\mathrm{y}$ & $\mathrm{n}$ & $\mathrm{CN}$ \\
\hline Virola elongata (Benth.) Warb. & $\begin{array}{l}\text { Tsempu, Sangre } \\
\text { de Gallo }\end{array}$ & 15 & M & $\mathrm{t}$ & $y$ & $\mathrm{n}$ & CN FR \\
\hline \multicolumn{8}{|l|}{ MYRSINACEAE } \\
\hline $\begin{array}{l}\text { Cybianthus sprucei } \\
\text { (Hook f.) A. Agostini }\end{array}$ & $\begin{array}{l}\text { Uchich Putush, } \\
\text { Putush }\end{array}$ & 7 & M & trlt & $y$ & $\mathrm{n}$ & ME \\
\hline \multicolumn{8}{|l|}{ NYCTAGINACEAE } \\
\hline Guapira sp. & Katsua & 74 & $S$ & $\mathrm{t}$ & - & $\mathrm{n}$ & None \\
\hline \multicolumn{8}{|l|}{ POACEAE } \\
\hline Coix lacryma-jobi L. & Trigo Tropical & 131 & $\mathrm{Ag}$ & $\mathrm{h}$ & $\mathrm{n}$ & $\mathrm{y}$ & FR \\
\hline Saccharum officinarum L. & $\begin{array}{l}\text { Numpapat, Pat, } \\
\text { Cana }\end{array}$ & 147,148 & $\mathrm{Ag}$ & $\mathrm{h}$ & $\mathrm{n}$ & y & FO \\
\hline
\end{tabular}




\begin{tabular}{|c|c|c|c|c|c|c|c|}
\hline Taxa & Common name(s) & Voucher & $\begin{array}{c}\text { Forest } \\
\text { Type }^{1}\end{array}$ & Habit $^{2}$ & Status $^{3}$ & Cult? ${ }^{4}$ & Uses $^{5}$ \\
\hline \multicolumn{8}{|l|}{ RUBIACEAE } \\
\hline Indet. 1 & Murak & 28 & $\mathrm{Ag}$ & $\mathrm{h}$ & - & $\mathrm{y}$ & FU \\
\hline Indet. 2 & Nashum & 37 & $\mathrm{Ag}$ & $\mathrm{h}$ & - & $\mathrm{y}$ & $\mathrm{PE}$ \\
\hline Indet. 3 & Waa & 11 & $M$ & $\mathrm{~h}$ & - & $\mathrm{n}$ & FR MI \\
\hline \multicolumn{8}{|l|}{ RUTACEAE } \\
\hline $\begin{array}{l}\text { Citrus aurantifolia } \\
\text { (Christm.) Swingle }\end{array}$ & Toronja & 130 & $\mathrm{Ag}$ & $\mathrm{t}$ & $n$ & $\mathrm{y}$ & FO \\
\hline Citrus limonum Risso & Narank, Naranja & 153 & $\mathrm{Ag}$ & $\mathrm{t}$ & $n$ & $\mathrm{y}$ & $\mathrm{FO}$ \\
\hline \multicolumn{8}{|l|}{ SAPOTACEAE } \\
\hline $\begin{array}{l}\text { Chrysophyllum } \\
\text { argenteum Jacq. }\end{array}$ & Yaas, (Wakam) & 33 & $\mathrm{Ag}$ & $\mathrm{t}$ & y & $\mathrm{n}$ & ME \\
\hline Pouteria baehniana Monach. & Yaas & 32,44 & $\mathrm{M}$ & $\mathrm{t}$ & $\mathrm{y}$ & $\mathrm{n}$ & $\begin{array}{l}\text { CN CR } \\
\text { FO FR } \\
\text { FU } \\
\end{array}$ \\
\hline $\begin{array}{l}\text { Pouteria caimito (Ruiz } \\
\text { \& Pav.) Radlk. }\end{array}$ & Yaas, Caimo & 133 & $\mathrm{Ag}$ & $\mathrm{t}$ & $\mathrm{y}$ & $\mathrm{y}$ & $\mathrm{FO}$ \\
\hline $\begin{array}{l}\text { Pouteria cuspidate } \\
\text { (A. DC.) Baehni }\end{array}$ & $\begin{array}{l}\text { Shaka, Shaka } \\
\text { Yaas, Tochi }\end{array}$ & 71 & $\mathrm{~S}$ & $\mathrm{t}$ & - & $\mathrm{n}$ & FO FR \\
\hline $\begin{array}{l}\text { Pouteria torta subsp } \\
\text { tuberculata (Sleumer) } \\
\text { T.D. Penn }\end{array}$ & $\begin{array}{l}\text { Kamputin Yaas, } \\
\text { Caimo de monte }\end{array}$ & 126 & $\mathrm{Ag}$ & $\mathrm{h}$ & $\mathrm{y}$ & $y$ & FR \\
\hline \multicolumn{8}{|l|}{ SOLANACEAE } \\
\hline $\begin{array}{l}\text { Brugmansia insignis (Barb. } \\
\text { Rodr.) Lockwood ex E.W. Davis }\end{array}$ & $\begin{array}{l}\text { Waimiatai Maikua, } \\
\text { Floripondio }\end{array}$ & 122 & $\mathrm{Ag}$ & s/trlt & hybrid & y & RM \\
\hline $\begin{array}{l}\text { Solanum endopogon } \\
\text { (Bitter) Bohs }\end{array}$ & $\begin{array}{l}\text { Takup, Tomate } \\
\text { de Arbol }\end{array}$ & 121 & $\mathrm{Ag}$ & $\mathrm{s}$ & y & y & FO \\
\hline $\begin{array}{l}\text { Solanum grandiflorum } \\
\text { Ruiz \& Pav. }\end{array}$ & Kukuch, Naranjilla & $125 b$ & $\mathrm{Ag}$ & s & y & y & FO \\
\hline Solanum mammosum L. & Chiam Tsuak & 136 & $\mathrm{Ag}$ & $\mathrm{h} / \mathrm{sbs}$ & $\mathrm{y}$ & $\mathrm{y}$ & $\mathrm{Ml}$ \\
\hline Solanum sessiliflorum Dunal & Kukuch, Naranjilla & $125 a$ & $\mathrm{Ag}$ & $\mathrm{s}$ & $\mathrm{y}$ & $\mathrm{y}$ & FO \\
\hline \multicolumn{8}{|l|}{ STERCULIACEAE } \\
\hline Theobroma subincanum Mart. & Wakamp, Wakam & 25,76 & $\mathrm{M}, \mathrm{S}$ & $\mathrm{t}$ & $\mathrm{y}$ & $n$ & FO FU \\
\hline \multicolumn{8}{|l|}{ VOCHYSIACEAE } \\
\hline Indet. 1 & Jea Paunim & 34 & $\mathrm{M}$ & $\mathrm{t}$ & - & - & $\mathrm{CN}$ \\
\hline \multicolumn{8}{|l|}{ ZINGIBERACEAE } \\
\hline $\begin{array}{l}\text { Renealmia alpinia } \\
\text { (Rottb.) Mass }\end{array}$ & Cumpia, Combia & 146 & $\mathrm{Ag}$ & $\mathrm{h}$ & y & Y & FO \\
\hline Renealmia sp. & Combia & 127 & $\mathrm{Ag}$ & $\mathrm{h}$ & - & $\mathrm{Y}$ & $\mathrm{FO}$ \\
\hline \multicolumn{8}{|l|}{ INDET A } \\
\hline- & Japa Iniai, Japajap & 31 & $M$ & $\mathrm{t}$ & - & - & $\mathrm{PE}$ \\
\hline
\end{tabular}

1 Forest Types: $M=$ mature forest, $S=$ =secondary forest, $A g=$ swidden area; 2 Plant Habits: $t=t r e e, s=s h r u b$, sbs=subshrub, h=herbaceous, v=vine; 3 Status: $y=$ Native species, $n=$ Non-native species; 4 Cultivation status: $y=c u l t i v a t e d$, $\mathrm{n}=$ =non-cultivated; 5 Uses: $\mathrm{CN}=$ construction, $\mathrm{CR}=$ craftsmanship, $\mathrm{DP}=$ dye and painting, $\mathrm{FO}=$ food, $\mathrm{FP}=$ food prep, $\mathrm{FR}=$ forage, $\mathrm{FU}=$ fuel, $\mathrm{ME}=$ medicine, $\mathrm{Ml}=$ miscellaneous, $\mathrm{PE}=$ personal, $\mathrm{RM}=$ ritual and magic, $\mathrm{TO}=$ tools. 


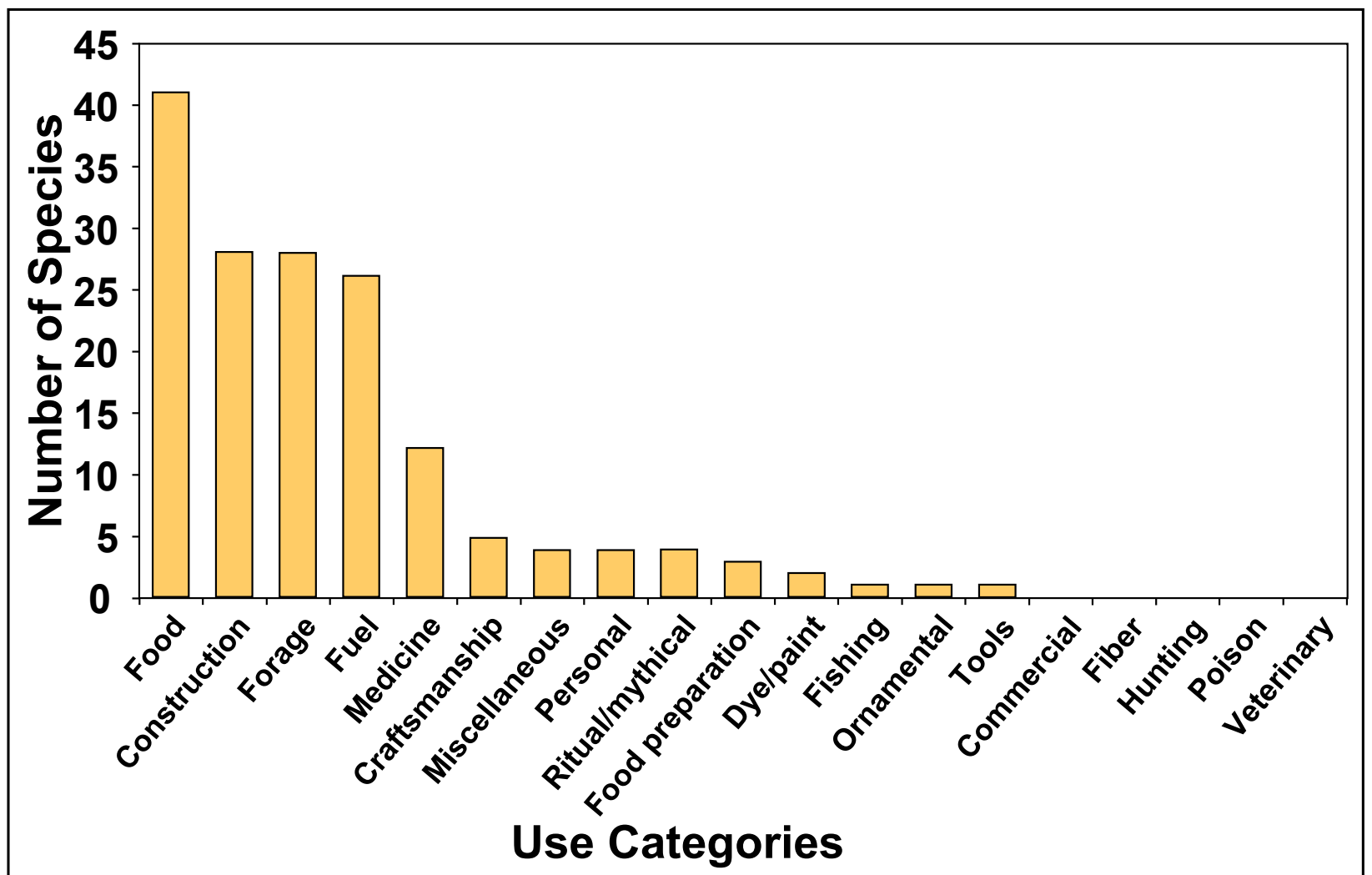

Figure 3. Number of species reported by use category for Shuar in Warints.

cies of cultivated and non-cultivated plants were collected in the $100 \mathrm{~m} 2$ swidden plot, which represent 28 genera and 20 families. Within the larger agricultural area, ten native tree species (Dacroydes peruviana (Loes.) J.F. Macbr., Piptocoma discolor (Kunth) Pruski, Bactris gasipaes Kunth, Bixa orellana L., Carica microcarpa Jacq., Dalbergia frutescens L.f., Inga densiflora Benth., Inga sapindoides Willd., Pouteria caimito (Ruiz \& Pav.) Radlk., Pouteria torta subsp. tuberculata (Sleumer) T.D. Penn) were protected by the landholder upon clearing and included in the collections.

Plants collected in the two forest transects and in the swidden area were categorized into the following uses: medicinal, food, forage, construction, fuel, craftsmanship, food preparation, dye/paint, fishing, ritual/magic, personal, tools and miscellaneous. The number of species in each category ranges from 1 to 41 . The use categories that contain the greatest number of plant species are food (41), construction (28), forage (28) and fuel (26), followed by medicine (12), craftsmanship (5), miscellaneous (4), personal (4), ritual/magic (4), food preparation (3), dye/ paint (2), fishing (1), ornamental (1) and tools (1) (Figure 3).

In comparing ethnobotanical data collected in mature versus secondary forest study sites, there were nearly equal numbers of ethnobotanically-useful trees collected in Transect A (34) and Transect B (33), with a slightly higher number of useful tree species in the mature forest $(91 \%)$ versus secondary forest transect $(85 \%)$ each having multiple uses (Figure 4). Neotropical secondary forests are typically richer in ethnobotanically-useful species than mature forests in the same locality, particularly in medicinal species (Chazdon \& Coe 1999, Kohn 1992, Voeks 1996). However, this was barely supported by the ethnobotanical research presented here, which yielded minimal medicinal data. The overall high percentage of tree species considered useful by the Shuar in Warints is notable in both transects (Table 4).

Table 4. Number of tree species collected and used in forest transects by the Shuar in Warints.

\begin{tabular}{|l|c|c|}
\hline \multirow{2}{*}{} & \multicolumn{2}{|c|}{ Forest Transect } \\
\cline { 2 - 3 } & Mature & Secondary \\
\hline \# species collected & 36 & 35 \\
\hline \# species used by Shuar & 33 & 30 \\
\hline$\%$ tree species used & $92 \%$ & $86 \%$ \\
\hline
\end{tabular}

Based on observation of Table 2, the families Fabaceae and Euphorbiaceae held the greatest number of ethnobotanically-useful species, with 15 and 8 respectively. Five other families collected, Solanaceae, Moraceae, Melastomataceae and Sapotaceae had 5-6 species in them that are used by the Shuar in Warints. Arecaceae, generally one of the dominant plant families in ethnobotanical 


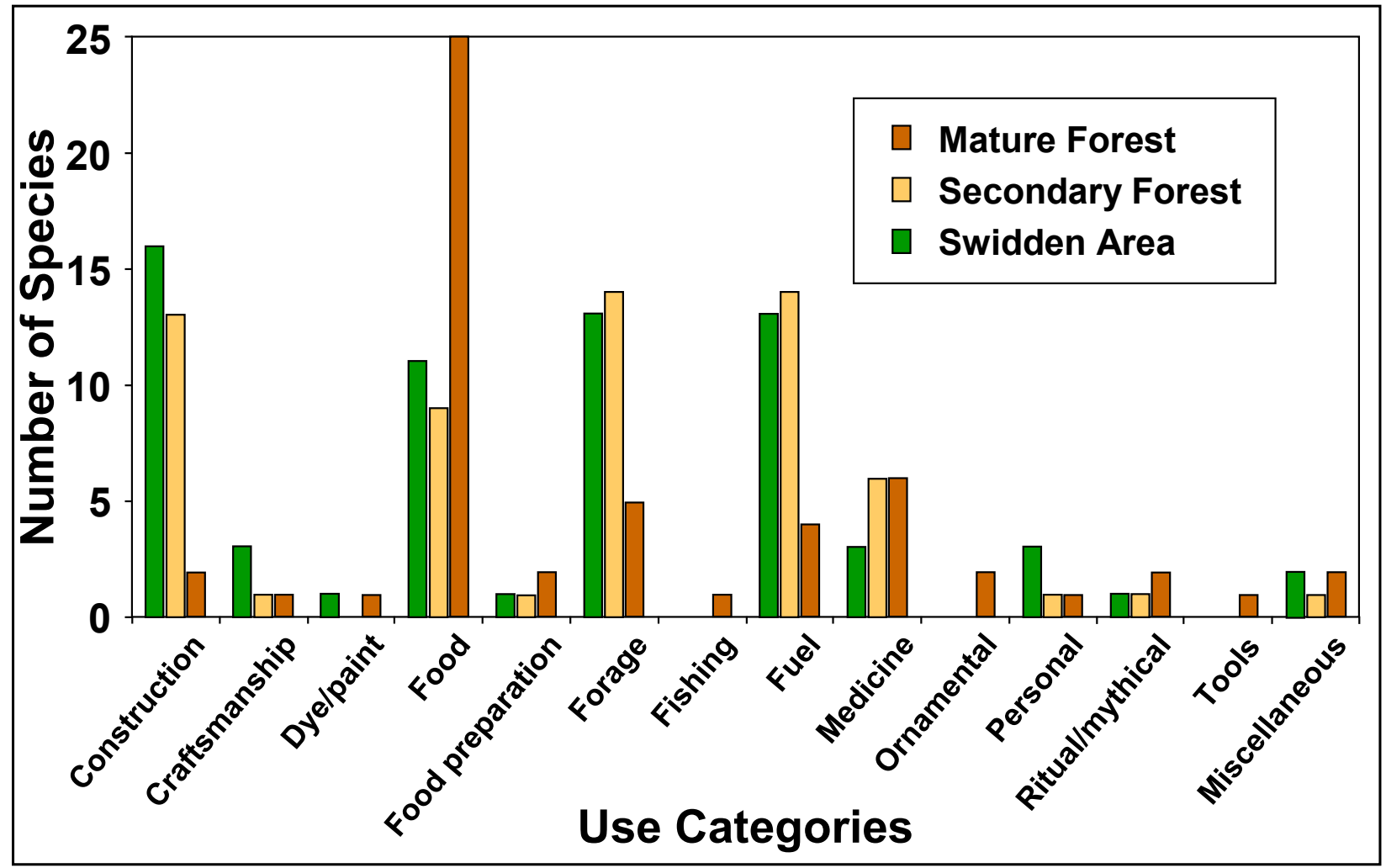

Figure 4. Number of plant species found in each use category reported by Shuar in Warints compared between mature forest, secondary forest and the swidden area.

studies in neotropical forests (Bennett et al. 2002, Chazdon \& Coe 1999), was essentially absent from this study, most likely due to the method of collection. Useful palms were not sought out, but instead collected only when they were found growing in the two forest transects or swidden plot. Two commonly used palms were included in the collections from the forest transects: Wettinia maynensis Spruce, terén in Shuar and Geonoma undata Klotzsch, kapanak in Shuar. W. maynensis was found growing abundantly in a section of the mature forest study site in which increased light was able to enter the canopy; it is considered useful in constructing Shuar homes (trunks as house posts, fronds for the roofs). As pictured, the fibers of $G$. undata are collected by women in Warints for later use in craftsmanship, primarily the construction of baskets (Figure 5). A third palm, Bactris gasipaes, uwi or chonta

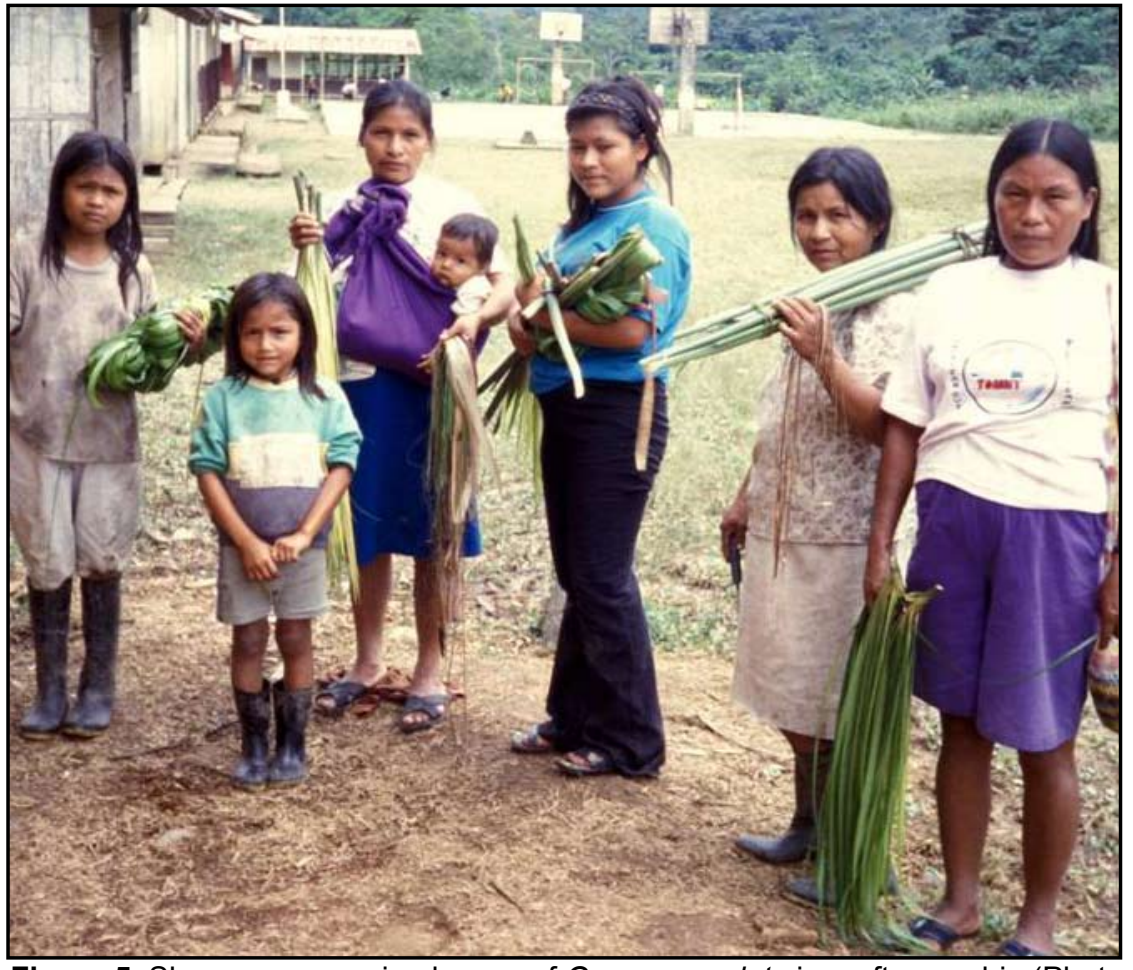

Figure 5. Shuar women using leaves of Genoma undata in craftsmanship (Photo by $A$. Duchelle). 


\section{Duchelle - Observations on Natural Resource Use and Conservation by the Shuar in Ecuador's Cordillera del Cóndor}

was collected in the agricultural plot. $B$. gasipaes is intensively cultivated by the Shuar, largely because the palm heart and cooked fruits are eaten or used in fermented beverages. Even though it was classified only under the food category of use in Warints, this palm is known to be used by the Shuar throughout Amazonia in manufacturing blowguns and lances, as well as using the trunks as posts in traditional Shuar houses (Bennett et al. 2002).

Nearly half of the ethnobotanically-useful plants (43) are considered to have multiple uses. Two species, Dacryodes peruviana, kunchái or copal, and Pouteria baehniana Monach., yaas, are found in five categories each. Ficus sp., wampu, is employed in four. Fourteen other species collected have three ethnobotanical uses according to the people of Warints. D. peruviana is employed in five use categories: construction, fuel, food, forage and personal. Notably, the resin from this tree is used as a candle substitute. One sample from this tree was misidentified by several informants, being called chipia (Protium fimbriatum Swart), which is another species in the Burseraceae family whose latex is also used as a candle substitute. D. peruviana is also reported used in the fishing category, because the resin of this tree is said to provide light on night fishing excursions (Bennett et al. 2002). This tree is referenced for its use in creating torches that were used by the Shuar in 1599 during the uprising that drove the Spanish out of their territory (Bennett et al. 2002, Harner 1972). The other tree with the greatest amount of uses in Warints, $P$. baehniana, yaas or washi yaas in Shuar, was not collected in study carried out by Bennett and his colleagues, but Pouteria caimito, also yaas or caimito, was found in both regions. $P$. caimito is cultivated throughout Amazonia for its appetizing fruits (Bennett et al. 2002, Vickers \& Plowman 1984).

\section{Observations on main plant use categories}

The information gathered from the ethnobotanical study in Warints, which focused on woody taxa and cultivars, contributes to a general understanding of Shuar forest use and swidden agricultural in this region and could prove valuable in future management decisions towards conservation of natural resources in the Cordillera del Cóndor. The information gathered in this study supports many of the findings in the large-scale study of the Shuar in the Cordillera del Cutucú (Bennett et al. 2002), particularly in paralleling the main categories of plant use observed in Warints - food, forage, construction and fuel.

\section{Food and forage}

A total of 41 species collected were employed in the food category, generally for use of the plants' fruits or roots. Of those collected, 28 were considered important for foraging animals, with nearly half of these (11) overlapping with the food category (cf. Bennet et al. 2002). In the forest transects alone, 20 species were considered food sources, highlighting the importance of the forest environment as a source of food. When discussing ethnobotany of the Shuar, it is essential to include the extensive cultivation and use of Manihot esculenta, commonly known as manioc, yucca or mama, which is the main staple of the Shuar diet. The roots are eaten daily and made into chicha, the fermented beverage which is also an essential part of daily Shuar life (Figures $6 \& 7$ ). The vast majority of land cleared in Warints is dedicated to the cultivation of this plant with other crops interspersed.

The diversity of plants collected in the swidden area demonstrates an emphasis on a multiple-cropping system with trees and crops interspersed. Much research has been done on how such cultivation methods enhance ecological integrity when compared with monoculture planting in tropical landscapes (Barton 1994, Denevan et al. 1984, Long \& Nair 2005, Posey 1985, Sirois et al.1998). The Shuar landholder of the area was cognizant of this, stating that the main reason he protected multiple large trees when clearing mature forest - many of which produce edible fruits for animals and/or humans - was primarily to attract wildlife, as well as provide food for his family

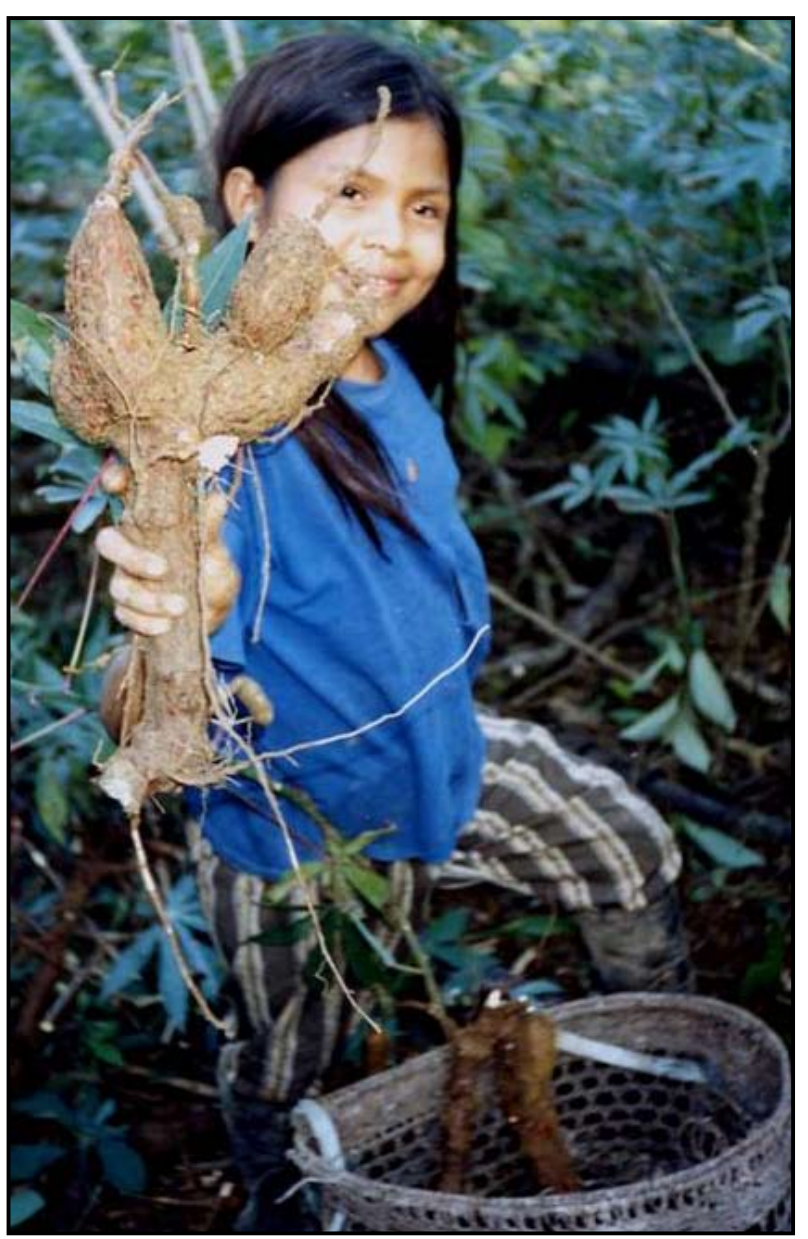

Figure 6. Shuar girl harvesting Manihot esculenta (Photo by $A$. Duchelle). 


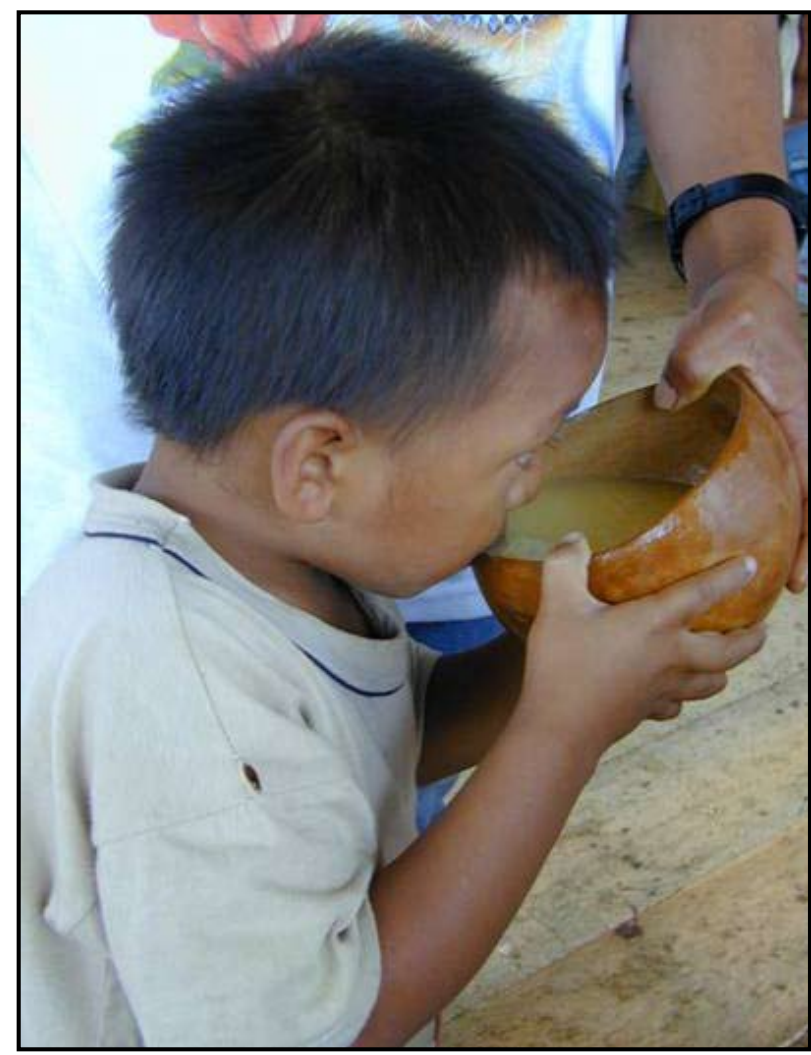

Figure 7. Shuar boy drinking chica made from Manihot esculenta (Photo by A. Duchelle).

and maintain soil structure. Bennett and his colleagues (2002:32) also noted the protection of plants by the Shuar in the Cordillera del Cutucú region when clearing mature forest for swidden areas and pastures, listing 155 species of plants that are protected for their use as forage, food or medicine.

\section{Construction and fuel}

Many of the trees collected in the mature and secondary forest transects were considered in subsistence use, particularly in the construction of traditional Shuar houses and as fuelwood. Interestingly, few of the finest hardwoods in Amazonia, generally found the family Meliaceae, were seen in Warints, primarily because the slopes of the Cordillera del Cóndor are higher in elevation and have a more montane floristic composition than the Amazon foothills and lowlands. Cedro (Cedrela odorata L.) was mentioned as existing in the vicinity, although it was not collected in the areas sampled. Legal and illegal logging are persistent in parts of the Shuar territory that are more easily accessed by roads. The lack of valuable hardwoods in the Cordillera del Cóndor is significant in that increased access to the area may not lead to intensive logging, as seen in the neighboring, but lower elevation, Shuar community of Santiago, where valuable hardwoods such as Sweitenia macrophylla King, Guarea kunthiana A. Juss. and Cedrela odorata are abundant and heavily exploited. Additionally, since there is no road close to Warints, the sale of timber is not yet a viable option in this community. In this study, community members were asked whether or not they would be willing to harvest and sell timber from their land if the opportunity presented itself. Nearly twothirds of those interviewed $(61 \%)$ responded positively with more than half of these qualifying their positive response by adding that some sort of management plan would need to be in place.

\section{Medicine}

The medicinal use category is less significant in Warints in comparison with the study by Bennett and his colleagues (2002). This could be explained in several ways. First, most of the collections in the study discussed here were made in the mature and secondary forest transects and were of woody taxa, while the majority of medicinal plants in the earlier, broader study were herbaceous (Bennett et al. 2002). Second, none of the informants interviewed in the Warints study were over the age of 59 , and possibly medicinal uses of taxa were not well known. Phillips and Gentry (1993) found that among the Tambopata in the Peruvian Amazon, edible plants were well known throughout most of the population, whereas knowledge of construction and commercially useful plants developed around age 30 , and a broad range of medicinal plant knowledge was reserved to community elders. Lastly, medicinal uses could have been purposely concealed to protect intellectual property.

Even though ethnographers tie the use of particular plants to the importance of shamanism in Shuar culture (Bennett 1992, Bennett et al. 2002, Descola 1984, Harner 1972), there is no known shamanism still practiced in Warints, having largely been replaced by the tenets of the Salesian missionaries. According to community members, however, shamanism is still a large part of the Shuar culture in other communities throughout the Cordillera del Cóndor. Brugmansia insignis (Barb. Rodr.) Lockwood ex E.W. Davis is grown in most Shuar gardens and used for its hallucinogenic properties, and is a hybrid of Brugmansia versicolor Lagerh. and Brugmansia suaveolens L., the latter of which is considered by the Shuar in the cutucú to be the most dangerous hallucinogenic plant, leading to insanity if over-consumed (Bennett et al. 2002). Banisteriopsis caapi (Griseb.) Morton, commonly known as ayahuasca, or in Shuar as natem, was referred to by several residents of Warints for its role in shamanism and on spiritual journeys to sacred waterfalls in the vicinity, but was not observed in this study.

\section{Threats to conservation in the Cordillera del Cóndor as perceived by the Shuar}

Based on interviews and free-listing and ranking exercises with community members in Warints, there are sev- 


\section{Duchelle - Observations on Natural Resource Use and Conservation by the Shuar in Ecuador's Cordillera del Cóndor}

eral threats to the conservation of biological diversity that must be addressed by FISCH, the Shuar natural resource managers and supporting NGOs in the implementation of a Shuar Protected Area in the Cordillera del Cóndor. Most importantly, there is a need to address future encroachment of mining companies. Gold, copper and other mineral deposits are abundant in the Shuar territory. Extensive gold mining, in southern Ecuador, has been done informally by small-scale miners who lack the financial resources and information needed to encourage the use of finer extraction and processing techniques and pollution controls. The most severe impact of small-scale uncontrolled mining is the abundance of toxic pollutants, which enter local watersheds (Mol \& Ouboter 2004, Tarras-Wahlberg et al. 2000). There is also evidence for the social destruction caused by miners entering into communities, including increases in violence and the spread of malaria (Barbeiri et al. 2005, Bezerra et al. 1996), and sexually-transmitted diseases to local people (Faas et al. 1999). Lands that have been cleared for mining are left essentially bare and dramatically altered in their re-vegetation potential due to the fact that the soil is turned multiple times in order to secure mineral deposits (Peterson \& Heemskerk 2001).

Mining was considered by respondents to be the most destructive threat to the environment in Warints. The results of monitoring done by the Ecuadorian Proyecto de Desarrollo Minero y su Control Ambiental (PRODEMINCA 1999) show that mining in southeastern Ecuador has severely contaminated waterways with waste products of cyanide, mercury and other metals and metalloids. This has resulted in a decrease of faunal diversity, with no animal species present in several highly polluted stretches of river (Tarras-Wahlberg et al. 2000). Many residents of Warints commented on the decrease in abundance of fish in the Warints River once the copper mining company began working in the territory and persisting one year after the company had abandoned the mining camp. Several of the women in the community also attributed recent sicknesses in their children (including boils on the skin, weight loss and loss of hair) to pollution of river water by the mining company, which was subsequently consumed by the community.

When asked if they would like to see the mining company return to Warints, two-thirds of the Shuar interviewed $(67 \%)$ responded negatively. They listed the following reasons for not wanting the company's return:

- $\quad$ illnesses caused in children

- $\quad$ pollution of air and water by the chemicals used

- forest damage

- conflict within the community

- noise pollution

- harassment of Shuar women by miners from outside the community

- the fact that many children quit school to work for the company.
Notably, all females interviewed were adamantly against the return of the mining company. The men who supported its return justified their response with the fact that the mining company provided an important internal source of income that was needed to take care of their families (e.g., provide a better education for their children). There are very few labor opportunities in Warints, with the majority of people making a living through subsistence agriculture. Wages from mining far exceeded the few, specialized wage-labor opportunities in the community such as teaching or carpentry.

Although many Shuar in Warints are aware that large-scale mining would have deleterious environmental impacts and augment conflict within communities in the Cordillera del Cóndor, mineral deposits are clearly a valuable natural resource. In an activity in which valuable natural resources were free-listed by community members, mineral deposits were mentioned the most, along with waterfalls, reflecting dual economic and spiritual values (Figure 8). The pressing need for cash among the Shuar in Warints, particularly with the community's increasing exposure to an outside cash economy, must be weighed alongside ecological concerns. Villagers with greater financial resources can pay for their children to go to school, own cattle, travel to and from the larger Ecuadorian towns, and buy guns, pots and pans, medicine and clothing. In 1999, it was leaders from the centro of Warints who privately contracted with the copper mining company, dismissing the FICSH legislation that prohibits any agreements with mining, logging or petroleum companies on Shuar lands. An alternative, more sustainable source of income in the community is clearly needed.

Another lesser threat to the environment, indicated by those interviewed in this study, is conflict over landholdings, both between and within communities in the Coangas Valley. As mentioned earlier, some families have clear titles to their land, while many do not. Fortunately, securing territorial control within the proposed Shuar Protected Area, considered essential for promoting conservation on indigenous lands (Brandon 1996, Schwartzman \& Zimmerman 2005), is a focus of the community environmental management plans currently supported by Fundación Natura and the Missouri Botanic Garden (Neill 2005).

Although hunting was not listed as a threat to the environment by those interviewed, it is clear from this study that more rigorous research is needed to determine the sustainability of subsistence hunting by the Shuar in the Cordillera del Cóndor. In a separate ranking exercise conducted in Warints, hunting was considered the most important resource-use activity by those interviewed (15 points), followed by forest protection (10), fishing (6) and the collection of wild plants (5). Animals commonly hunted in Warints include wild cracids (large game birds, including Chamaepetes goudotii, Penelope jacquacu and Pipile pipile), peccary (Tayassu pecari), paca (Agouti paca), 


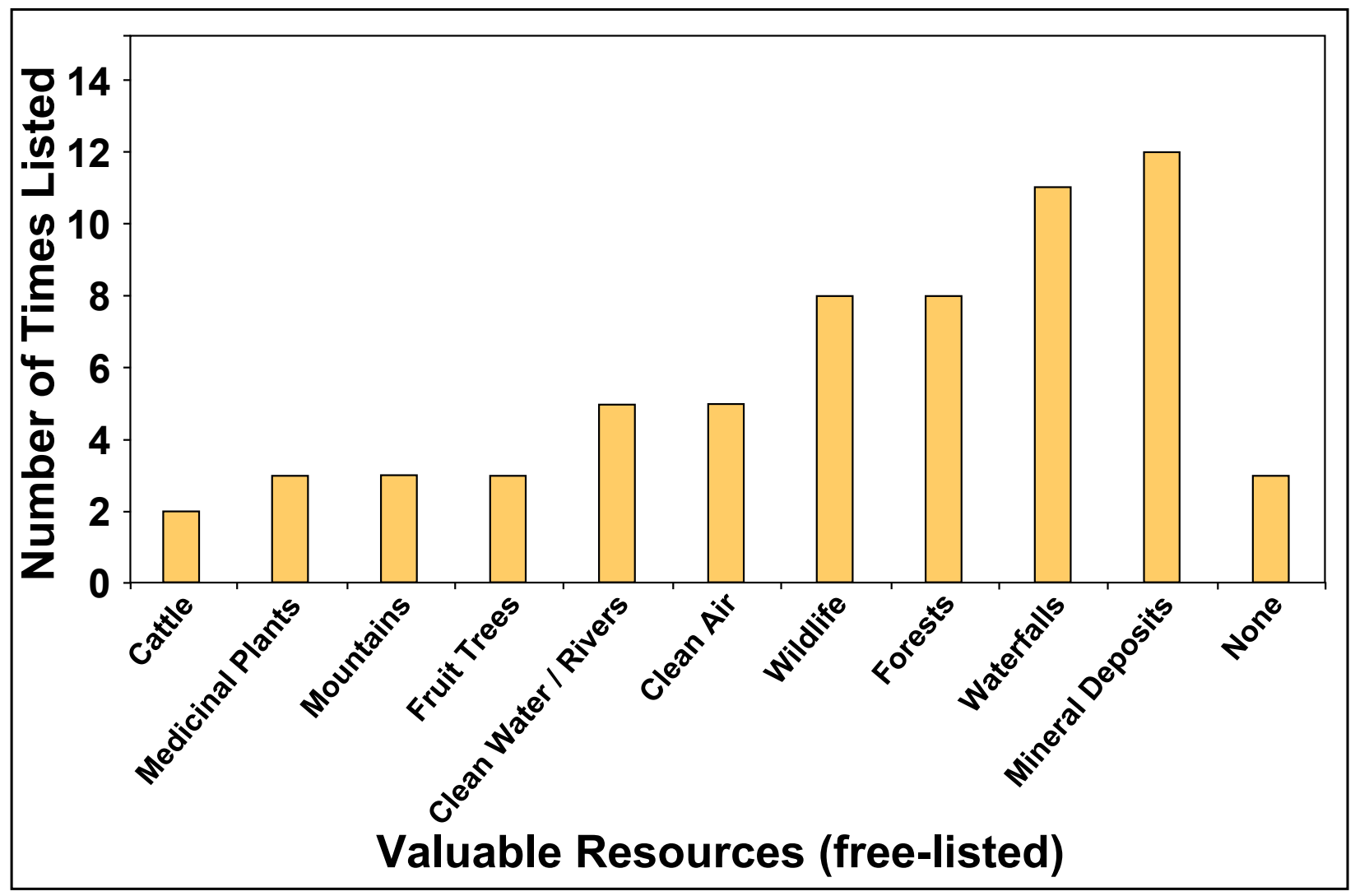

Figure 8. Valuable natural resources as free-listed by the Shuar in Warints.

armadillo (Cabassous unicinctus and Dasypus novemcinctus), agouti (Dasyprocta fuliginosa), a variety of other birds and occasionally tapir (Tapirus terrestris). In general, hunting provides indigenous people with their main source of protein and is also culturally significant among many of these groups, including the Shuar (Harner 1972, Vickers 1991). Large-bodied animals are selectively hunted throughout the Amazon lowlands and highlands, markedly changing the faunal composition and eventually affecting forest ecology (Peres 2000). In Warints, there has been a recent noticeable decline in abundance and diversity of wildlife among community members interviewed. The perceived scarcity of large-bodied animals in Warints can be also be inferred from noted profusion of small-bodied game species in the vicinity (Peres 2000, Robinson \& Redford 1986). Since traditional practices of using blowguns and barbasco, a plant-based toxin used in fishing, have evolved into firearms and dynamite, the dynamic of hunting and fishing in the region has changed and several community members linked these high-impact practices with the disappearance of wildlife in the area. People must travel much farther from the centro of Warints to find game. The effects of hunting on wildlife will need to be quantified in the region through further research before $\mathrm{FICSH}$, with the technical advice of the trained Shuar interns and supporting NGOs, implements and monitors regulations for effective wildlife management within the new Shuar Protected Area.

\section{Conclusions}

Understanding and valuing traditional and current Shuar knowledge systems and land use practices is an important aspect of promoting conservation in the Cordillera del Cóndor. This study provides insights into how one Shuar community in the region use forests, cultivate swidden land, value natural resources and view changes to the environment.

Based on Shuar knowledge and use of woody taxa observed in the mature and secondary forest transects of this study, it is clear that the use of forest products for subsistence is of great importance to people in Warints, although other traditional activities such as shamanism are no longer practiced there. The primary use categories for forest plants are construction, fuel, food and forage. In the swidden area, plants collected are primarily used for consumption and classified within the food category. Although a variety of woody taxa is protected when forest is cleared in swidden cultivation, primarily to attract wildlife, trees were not observed to be planted in Warints except in gardens immediately adjacent to homes. Maintaining habitat for wildlife, which includes trees used for 


\section{Duchelle - Observations on Natural Resource Use and Conservation by the Shuar in Ecuador's Cordillera del Cóndor}

forage, could be an important incentive for conservation in the Cordillera del Cóndor. However, hunting could greatly reduce abundance of larger mammals in cultivated areas (Naughton-Treves 2002), especially if this predation is not managed in a sustainable way. In Sangay National Park, located north of the Cordillera del Cóndor, the modification of traditional Shuar swidden agriculture through an NGO supported agroforestry initiative has resulted in less cleared land, but also in changing gender roles as men spend more time on cultivating crops with economic value. Interestingly, investment of time by Shuar men in new labor opportunities, including involvement in agroforestry systems, is considered the main reason for the decline in hunting in the area (Rosero 2004). There is potential to incorporate ethnobotanical knowledge of species protected by the Shuar when clearing forest into future development of agroforestry systems in the Cordillera del Cóndor, based on further research and collaborations. Additionally, a more thorough understanding and empowerment of Shuar culture, including how the Shuar use and manage forests, is essential if the region becomes more integrated into the market economy, and certain timber and non-timber forest products are potentially managed for sale.

Increased access to the region, such as the possible influx of large-scale mining operations and associated infrastructure in the Coangas Valley, has the potential to alter Shuar use and management of forests. In light of such change, it is necessary to promote collaborations between FISCH, Shuar natural resource managers, communities in the region, supporting NGOs and the Ecuadorian government for conservation to succeed in the Cordillera del Cóndor. Although FISCH has expressed its opposition to large-scale mining on Shuar lands, it is the Ecuadorian government who controls sub-soil rights and holds the power to decide whether the Cordillera del Cóndor will be opened to mining. As seen in this study, there are very negative environmental and social impacts that could occur if large-scale mining is introduced in the region. The proposed Shuar Protected Area could serve as a buffer in light of such changes, but has yet to be formally recognized by the Ecuadorian state (Neill 2005). A more regional, participatory environmental planning process, which includes indigenous leaders, trained Shuar natural resource managers, researchers from supporting NGOs and representatives from the Ecuadorian government could be a way to form policy that promotes both sustainable economic alternatives for local people and conservation of the Cordillera del Cóndor's unique ecosystem.

Since the Cordillera del Cóndor is of great interest to conservation biologists for its unique biological diversity, additional partnerships between the Shuar and conservationists, which simultaneously provide sustainable economic alternatives for communities and promote conservation could be explored, such as the creation of a biological research station as seen with the Kayapó in the eastern Brazilian Amazon (Zimmerman et al. 2001). FICSH and the
Shuar natural resource managers, supported by collaborations with non-governmental and governmental organizations, will need to explore such options as the struggle to balance the conservation of biodiversity with sustainable development continues in the Shuar Protected Area in Ecuador's Cordillera del Cóndor.

\section{Acknowledgements}

This study was financially supported by the University of Wisconsin Herbarium and a Travel Grant from the UW Gaylord Nelson Institute for Environmental Studies. I am most grateful to David Neill, for without his willingness and generosity in allowing my participation in the "Training and Development in conservation capacity for indigenous organizations in Ecuador" and facilitating my entrance into the Cordillera del Cóndor, this study would not have been possible. I would also like to thank Galo Zapata, Germán Toasa and Nixon Revelo for their extensive research assistance, along with the Shuar trainees, Camilo Kajekai, Tuntiak Katan, Gilberto Tsuink and Abel Wisum, as well as Socorro Casaluzan, Carlos Nastacuaz, Daniel Pay, and Jaime Shiguango. To the wonderful people of Warints who openly welcomed me into their community and into their homes, I am also indebted. A final thank you to Paul Berry, Lisa Naughton and Stanley Temple for their sound advice on earlier versions of this paper, along with one anonymous reviewer. I hold full responsibility for its limitations.

\section{Literature Cited}

Alexiades, M.N. \& J. Wood. 1996. Selected Guidelines for Ethnobotanical Research: A field manual. New York Botanical Garden Press, Bronx, NY.

Arambiza, E. \& M. Painter. 2006. Biodiversity conservation and the quality of life of indigenous people in the Bolivian Chaco. Human Organization 65:20-34.

Barbieri, A.F., D.O. Sawyer \& B.S. Soares. 2005. Population and land use effects on malaria prevalence in the southern Brazilian Amazon. Human Ecology 33:847-874.

Barton, D. 1994. Indigenous Agroforestry in Latin America: A blueprint for sustainable agriculture? National Resources Institute, Chatam, UK.

Bennett, B.C. 1992. Hallucinigenic plants of the Shuar and related indigenous groups in Amazonian Ecuador and Peru. Brittonia 44:483-493.

Bennett, B.C., M.A. Baker \& P. Gomez Andrade. 2002. Ethnobotany of the Shuar of Eastern Ecuador. New York Botanical Garden Press, Bronx, NY. 
Berkes, F., J. Colding \& C. Folke. 2000. Rediscovery of traditional ecological knowledge as adaptive management. Ecological Applications 10:1251-1262.

Berlin, B. 1992. Ethnobotanical Classification: Principles of categorization of plants and animals in traditional societies. Princeton University Press, Princeton, NJ.

Bezerra, O., A. Vérissimo \& C. Uhl. 1996. The regional impacts of small-scale gold mining in Amazonia. Natural Resources Forum 20:305-317.

Borman, R. 1999. Cofán: Story of the forest people and outsiders. Cultural Survival Quarterly 23:48-50.

Bottasso, J. 1976. El Cuerpo Humano, Animales y Plantas. Serie $\mathrm{A}, \mathrm{n}^{\circ} 1$. Mundo Shuar, Sucúa, Ecuador.

Brandon, K. 1996. Traditional peoples, nontraditional times: social change and the implications for biodiversity conservation. Pp. 219-236 in Traditional Peoples and Biodiversity Conservation in Large Tropical Landscapes. Edited by K.H Redford \& J.A. Mansour. The Nature Conservancy, Arlington, VA.

Chazdon, R.L. \& F.G. Coe. 1999. Ethnobotany of woody species in second-growth, old-growth, and selectively logged forests of northeastern Costa Rica. Conservation Biology 13:1312-1322.

Conklin, B.A. \& L.R. Graham. 1995. The shifting middle ground: Amazonian indians and eco-politics. American Anthropologist 97:695-710.

COICA. 1989. Two agendas on Amazon development. Cultural Survival Quarterly 13:75-87.

Descola, P. 1994. In the Society of Nature: A native ecology in Amazonia. Cambridge University Press, Cambridge, UK.

Denevan, W.M., J.M Treacy, J.B. Alcorn, C. Padoch, J. Denslow \& S.F. Paitan. Indigenous agroforestry in the Peruvian Amazon - Bora indian management of swidden fallows. Interciencia 9:346-357.

Dove, M. 2006. Indigenous people and environmental politics. Annual Review of Anthropology 35:191-208.

Fass, L., A. Rodríguez-Acosta \& G. Echeverría de Pérez. 1999. HIVISTD transmission in gold-mining areas of Bolivar State, Venezuela. Interventions for diagnosis, treatment and prevention. Revista Panamericana de Salud Publica 5:58-65.

Fearnside, P.M. 2003. Conservation in Brazilian Amazonia: Understanding the dilemmas. World Development 31:757-759.
Harner, M.J. 1972. The Jívaro: People of the sacred waterfalls. Natural History Press, Garden City, NY.

Huntington, H.P. 2000. Using traditional ecological knowledge in science: Methods and applications. Ecological Applications 10:1270-1274.

Kohn, E.O. 1992. Some observations on the use of medicinal plants from primary and secondary growth by the Runa of eastern lowland Ecuador. Journal of Ethnobiology 12:141-151.

Long, A.L. \& P.K.R. Nair. 2005. Trees outside forests: agro-, community, and urban forestry. New Forests 17:145-174.

McNeely, J.A. 2003. Biodiversity, war and tropical forests. Pp 1-20 in War and Tropical Forests: Conservation in areas of armed conflict. Edited by S.V. Price. The Haworth Press, Inc., Binghamton, NY.

Ministerio del Ambiente República del Ecuador. 2006. http://www.ambiente.gov.ec/

Mol J.H. \& P.E. Ouboter. 2004. Downstream effects of erosion from small-scale gold mining on the instream habitat and fish community of a small neotropical rainforest stream. Conservation Biology 18:201-214.

Nadasday, P. 1999. The politics of TEK: Power and the "integration" of knowledge. Arctic Anthropology 36:1-18.

Naughton-Treves, L. 2002. Wild animals in the garden: Conserving wildlife in Amazonian agroecosystems. Annals of the Association of American Geographers 92:488506.

Neill, D.A. 2005. Cordillera del Cóndor: Botanical treasures between the Andes and Amazon. Plant Talk 41:1721.

Nepstad, D., S. Schwartzman, B. Bamberger, M. Santilli, D. Ray, P. Schlesinger, P. Lefebvre, A. Alencar \& E. Prinz. 2006. Inhibition of Amazon deforestation and fire by parks and indigenous reserves. Conservation Biology 20:65-73.

Peres, C.A. 1994. Indigenous reserves and nature conservation in Amazonian forests. Conservation Biology 8:586-588.

Peres, C.A. 2000. Effects of subsistence hunting on vertebrate community structure in amazonian forests. Conservation Biology 14:240-253.

Peterson, G.D. \& M. Heemskerk. 2001. Deforestation and forest regeneration following small-scale gold mining in the Amazon: The case of Suriname. Environmental Conservation 28:117-126. 


\section{Duchelle - Observations on Natural Resource Use and Conservation by the Shuar in Ecuador's Cordillera del Cóndor}

Phillips, O. \& A.H. Gentry. 1993. The useful plants of Tambopata Peru: II. Additional hypothesis testing in quantitative ethnobotany. Economic Botany 47:33-43.

Posey, D.A. 1985. Indigenous management of tropical forest ecosystems: The case of the Kayapo indians of the Brazilian Amazon. Agroforestry Systems 3:139-158.

PRODEMINCA. 1999. Monitoreo Ambiental de las áreas Mineras en el sur del Ecuador Entre 1996-1998. Ministerio de Energía y Minas. Quito.

Redford, K. \& C. Padoch. 1992. Conservation of Neotropical Forests: Working from traditional resource use. Columbia University Press, New York, NY.

Robinson, J. \& K.H. Redford. 1986. Body size, diet, and population-density of neotropical mammals. American Naturalist 128:665-680.

Rosero, C. 2004. Women Hunters and Men Gathers: Gender role changes among the Shuar. M.A. thesis. University of Florida, Gainesville.

Rubenstein, S. 2002. Alejandro Tsakimp: A Shuar healer in the margins of history. University of Nebraska Press, Lincoln, NE.

Rudel, T.K., D. Bates \& R. Machinguiashi. 1999. Ecologically noble Amerindians? Cattle ranching and cash cropping among the Shuar and colonists in Ecuador. Latin American Research Review 37:144-159.

Salazar, E. 1981. The Federación Shuar and the Colonization Frontier. Cultural transformations and ethnicity in modern Ecuador. Edited by N.E. Whitten Jr. University of Illinois Press, Urbana, IL.

Schulenberg, T.S. \& K. Awbrey. 1997. The Cordillera del Condor region of Ecuador and Peru: A biological assessment. RAP Working Papers 7:1-231.

Shanley, P. \& N. Rosa. 2004. Eroding knowledge: An ethnobotanical inventory in Eastern Amazonia's logging frontier. Economic Botany 58:135-160.

Schwartzman, S. \& B. Zimmerman. 2005. Conservation alliances with indigenous peoples of the Amazon. Conservation Biology 19:721-727.

Sierra, R. 1999. Traditional resource-use systems and tropical deforestation in a multi-ethnic region in north-west Ecuador. Environmental Conservation 26:136-145.

Sirois, M.C., H.A. Margolis \& C. Camire. 1998. Influence of remnant trees on nutrients and fallow biomass in slash and burn agroecosystems in Guinea. Agroforestry Systems 40:227-246.
Tarras-Wahlberg, N.H., A. Flachier, G. Fredriksson, S. Lane, B. Lundberg \& O. Sangfors. 2000. Environmental impacts of small-scale and artisanal gold mining in southern Ecuador. Ambio 29:484-491.

Unicef. 2006. http://www.unicef.org/spanish/infobycountry/ecuador_statistics.html

Vickers, W.T. \& T. Plowman. 1984. Useful plants of the Siona and Secoya indians of eastern Ecuador. Fieldiana Botany 15:1-63.

Vickers, W.T. 1991. Hunting yields and game composition over ten years in an Amazon Indian territory. Pp. 53-81 in Neotropical Wildlife Use and Conservation. Edited by J.G. Robinson \& K.H. Redford. University of Chicago Press, Chicago, IL.

Voeks, R.A. 1996. Tropical forest healers and habitat preference. Economic Botany 50:381-400.

Zimmerman, B., C.A. Peres, J.R. Malcolm \& T. Turner. 2001. Conservation and development alliances with the Kayapó of south-eastern Amazonia, a tropical forest indigenous people. Environmental Conservation 28:1022. 
